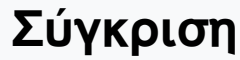

Tóp. 13 (2002)

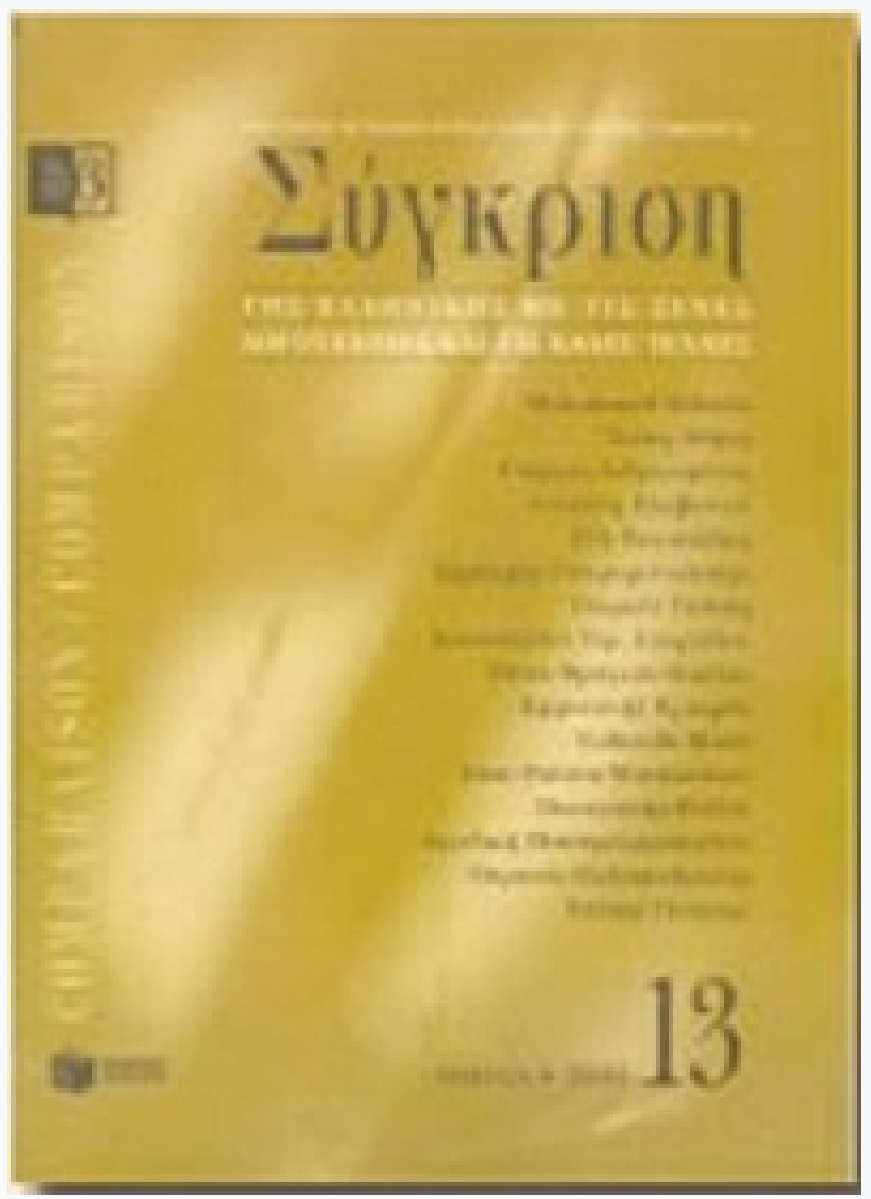

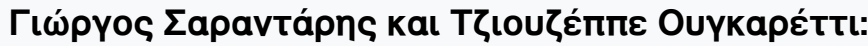

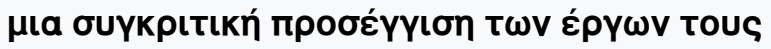

Gabriella Macrì

doi: $10.12681 /$ comparison.10713

Copyright $\odot$ 2016, Gabriella Macrì

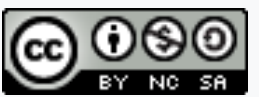

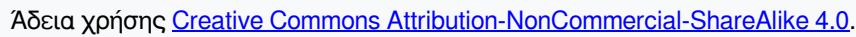

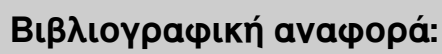

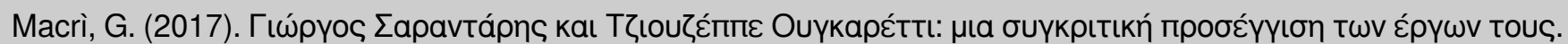

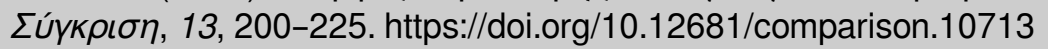




\author{
G A B R I E L L A M A C R Ì
}

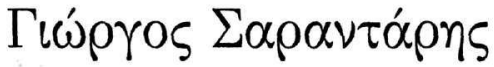

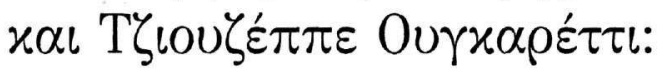

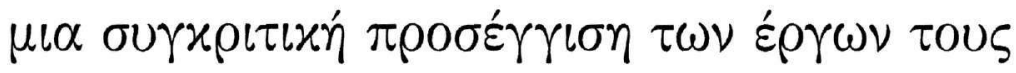

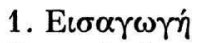

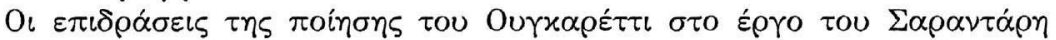

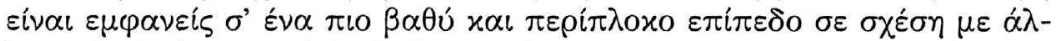

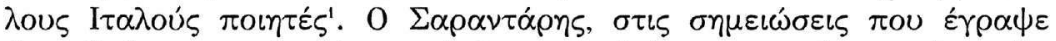

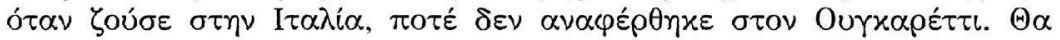

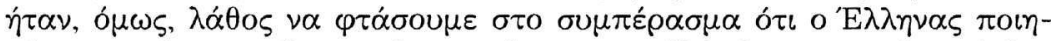

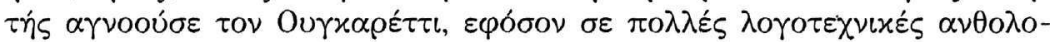

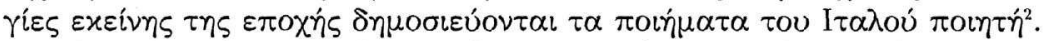

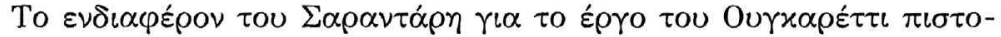

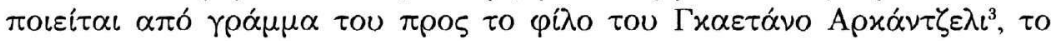

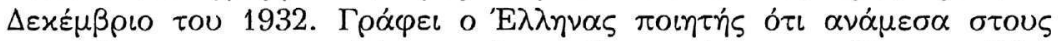

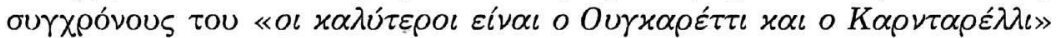

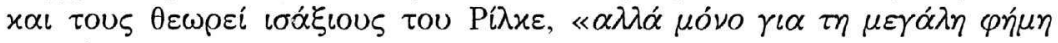

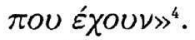

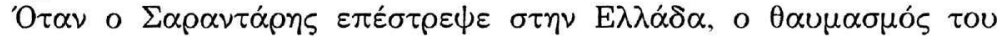

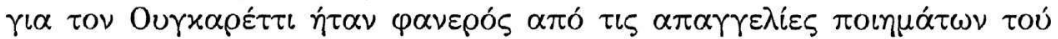

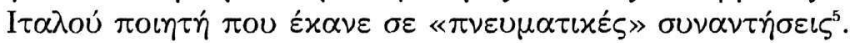

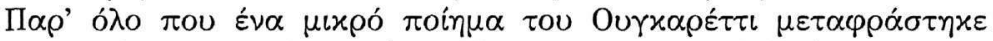

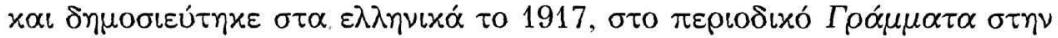

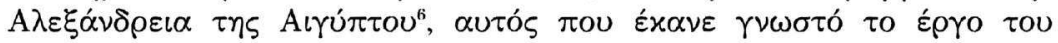

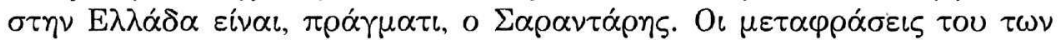

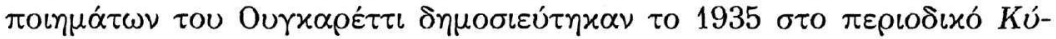

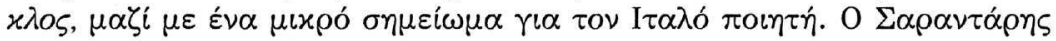

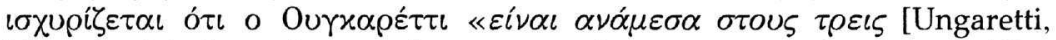
Montale, Saba] $x \alpha \tau \dot{\alpha} \tau \eta \nu \gamma \nu \omega ́ \mu \eta \mu \alpha \zeta, ~ o ~ \mu o ́ v o \zeta ~ \pi o v ~ \mu \pi o \rho \varepsilon i ~ \nu \alpha ~ \varepsilon i \pi \omega \theta \varepsilon i$ Ev-

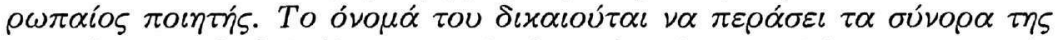

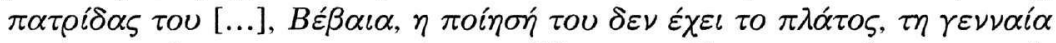

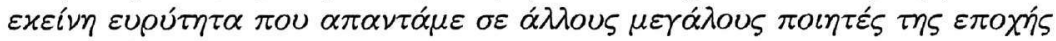

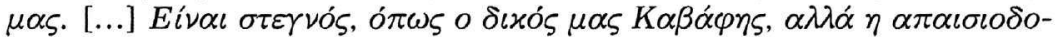

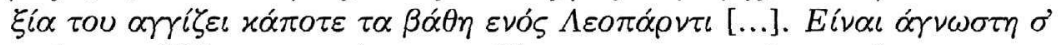

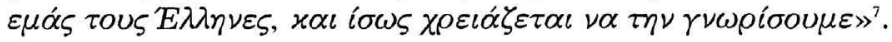




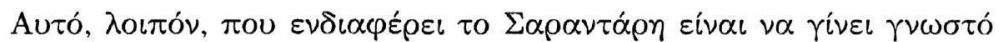

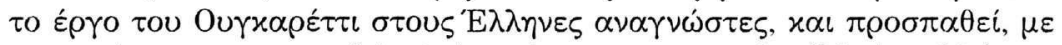

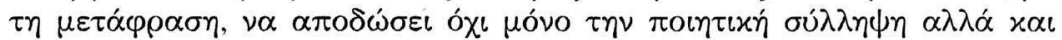

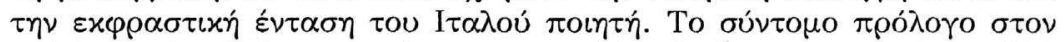

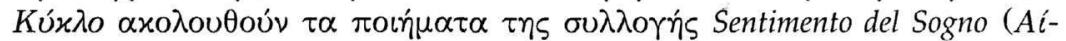

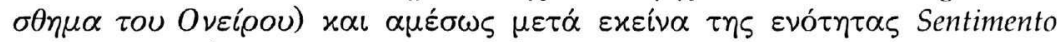

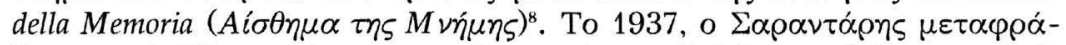

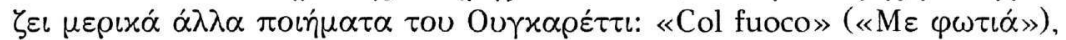

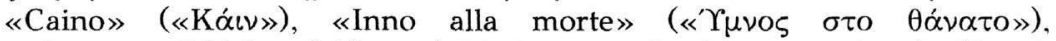

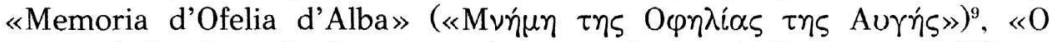

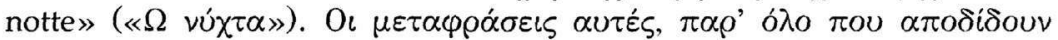

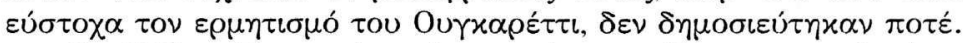

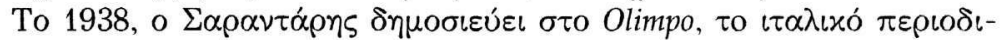

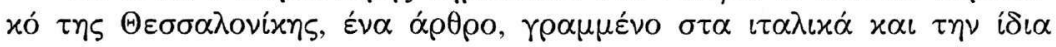

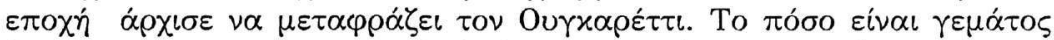

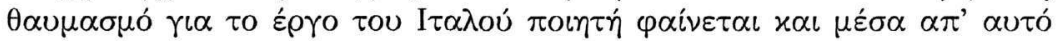

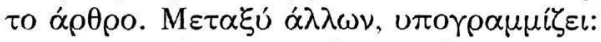

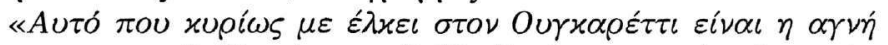

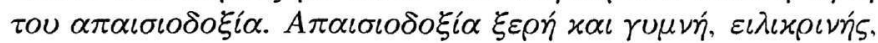

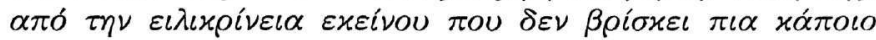

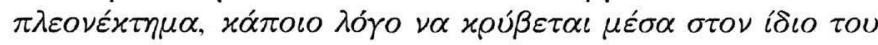

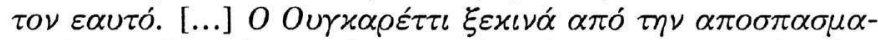

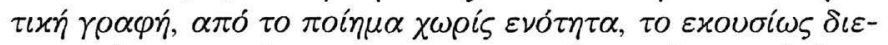

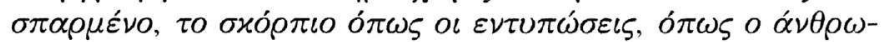

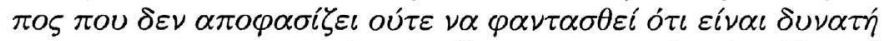

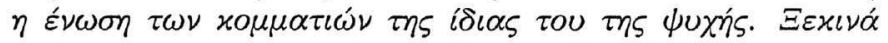

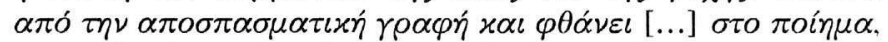

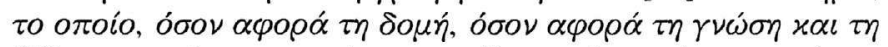

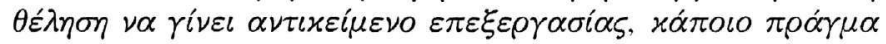

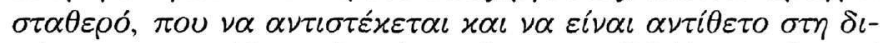

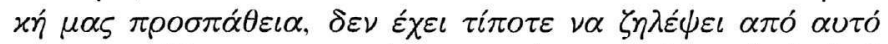

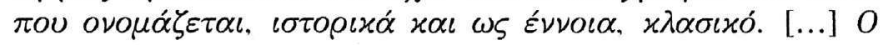

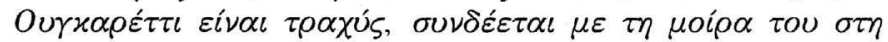

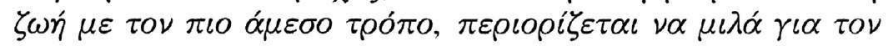

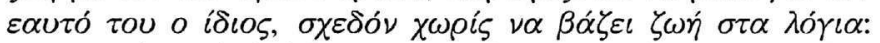

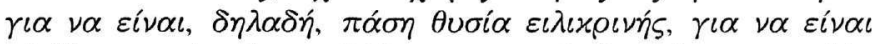

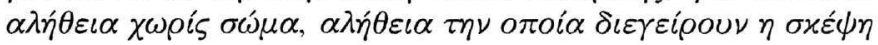

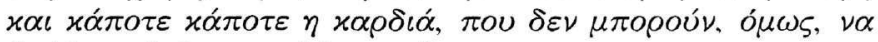

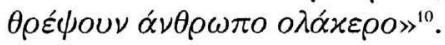

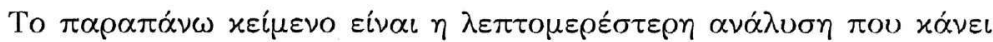

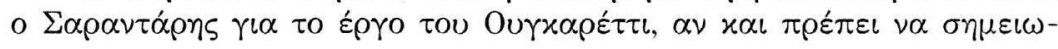




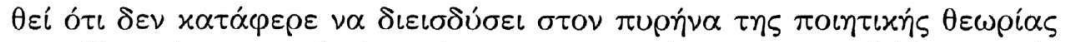

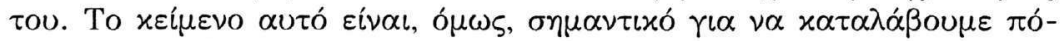

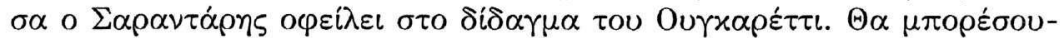

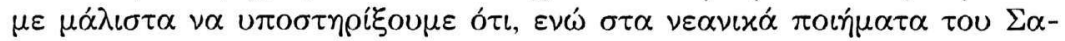

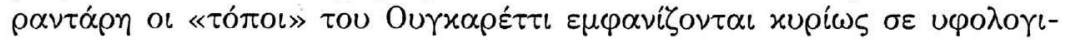

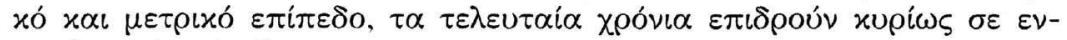

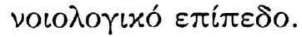

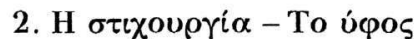

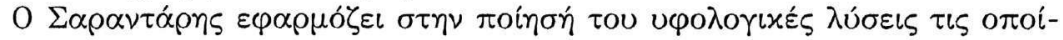

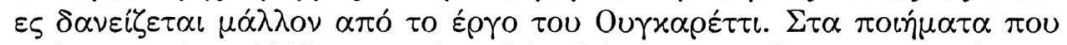

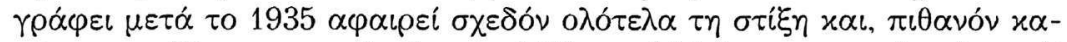

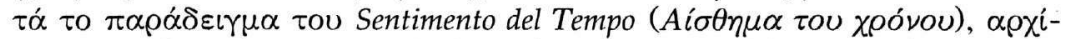

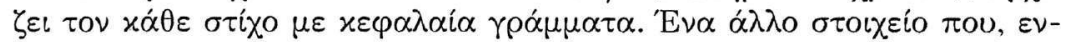

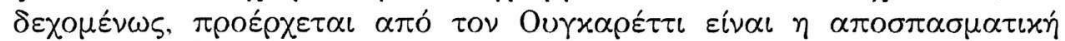

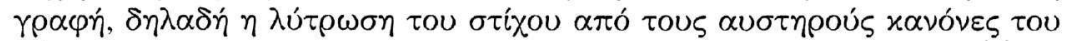
$\mu \varepsilon ́ \tau \rho o v$.

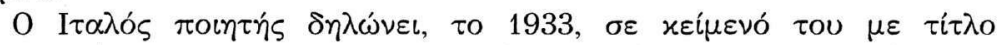

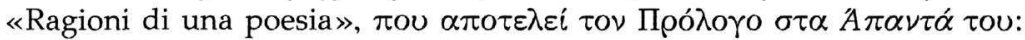

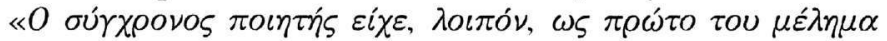

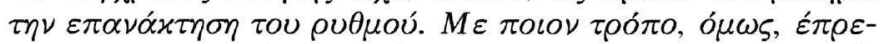

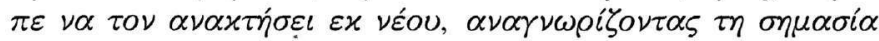

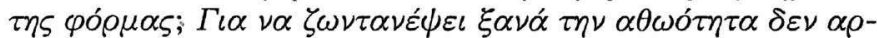

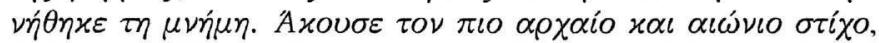

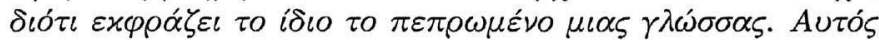

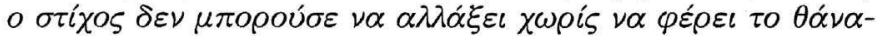

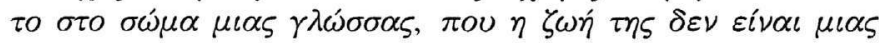

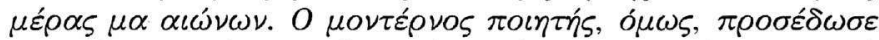

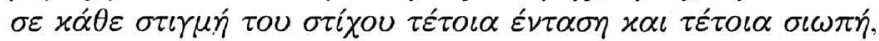

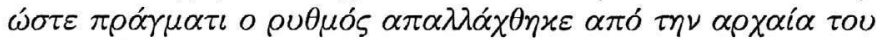

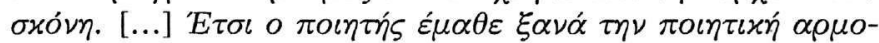

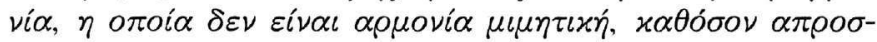

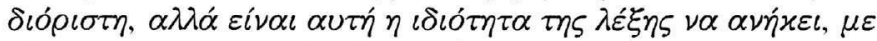

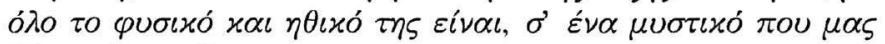

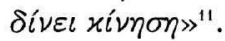

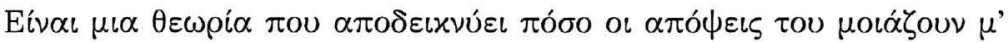

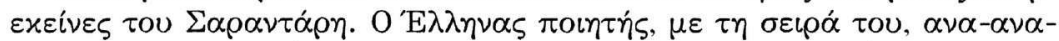

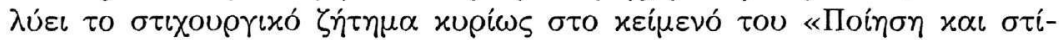

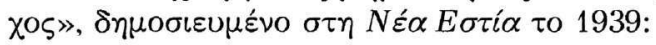




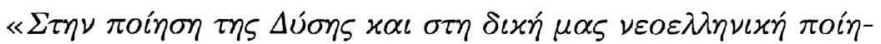

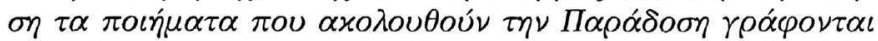

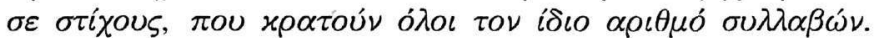

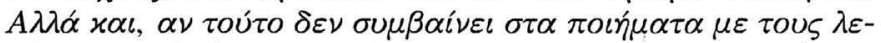

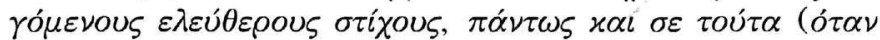

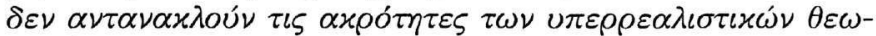

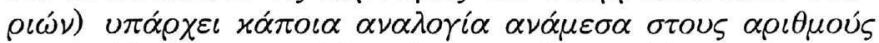

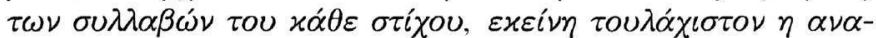

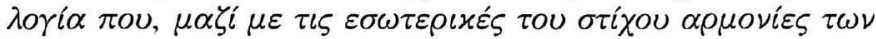

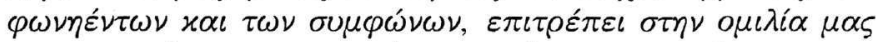
$\nu \alpha$ $\alpha \nu \dot{\eta}^{\prime} x \varepsilon(\gg)^{12}$.

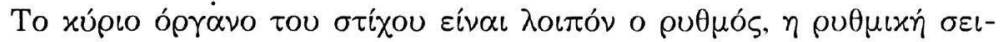

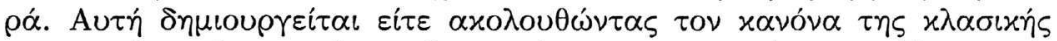

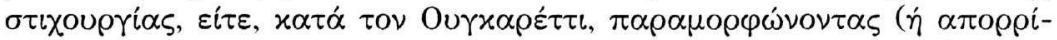

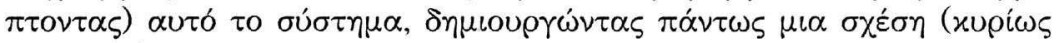

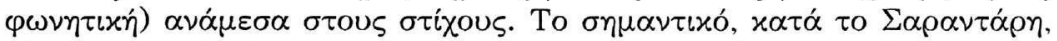

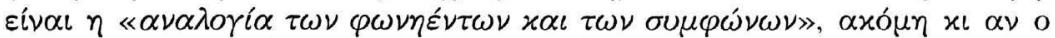

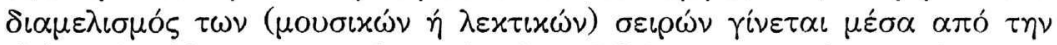

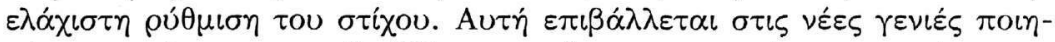

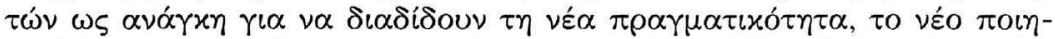

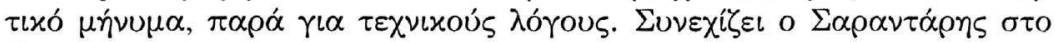

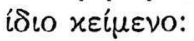

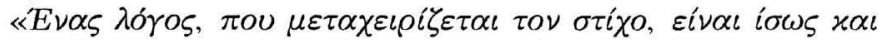

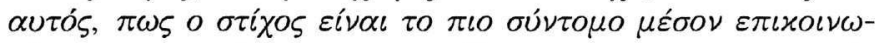

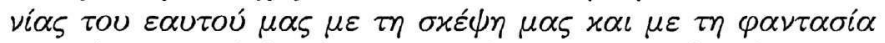

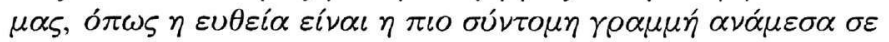

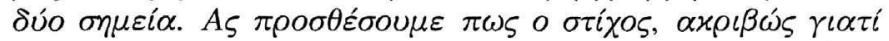

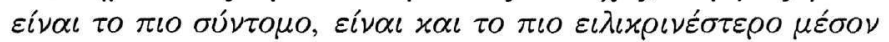

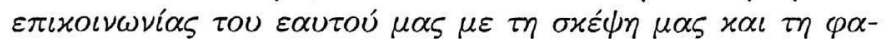

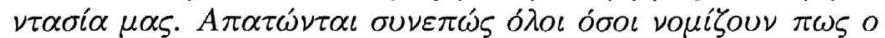

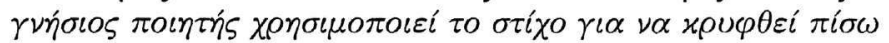

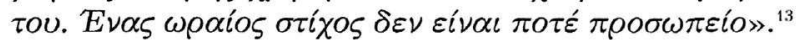

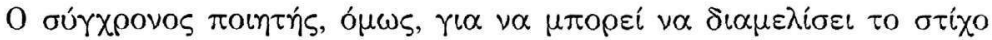

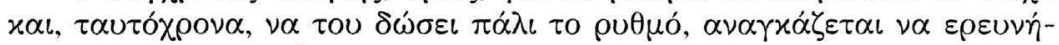

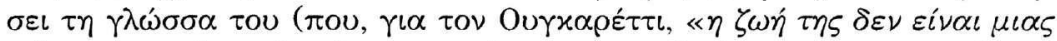

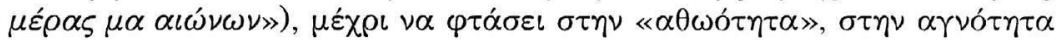

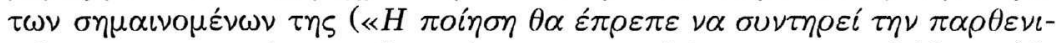

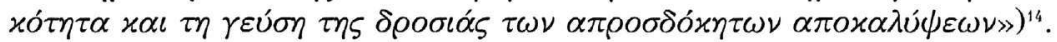

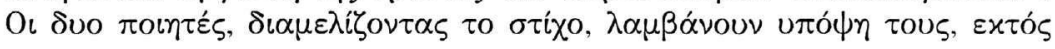




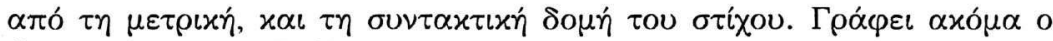

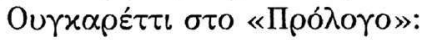

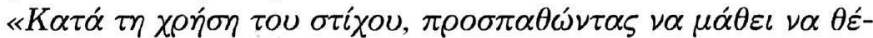

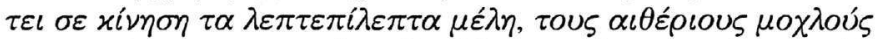

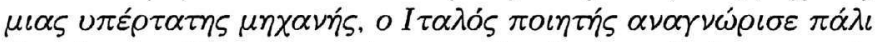

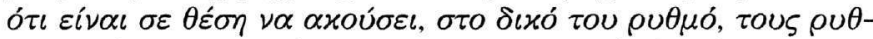

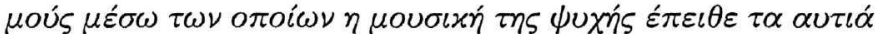

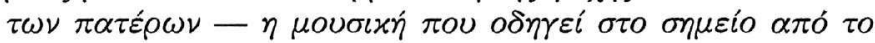

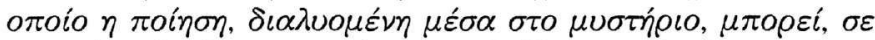

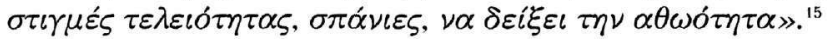

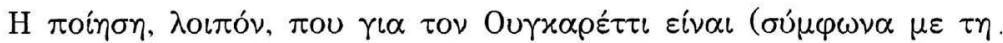

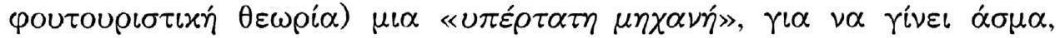

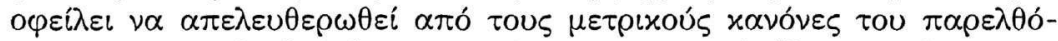

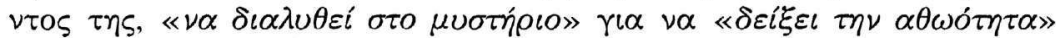

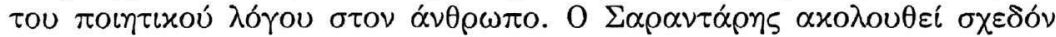

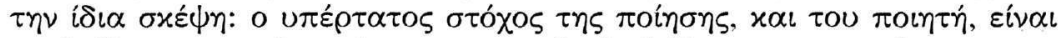

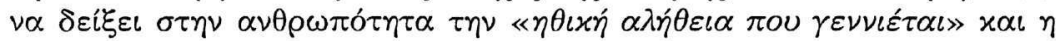

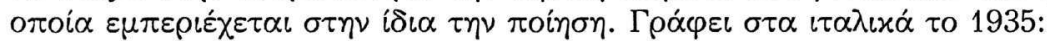

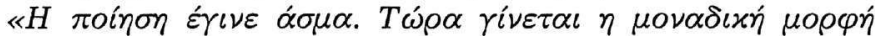

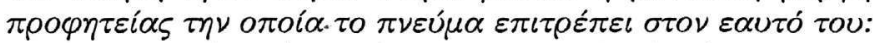

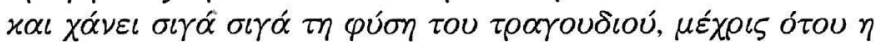

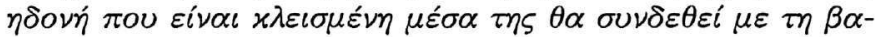

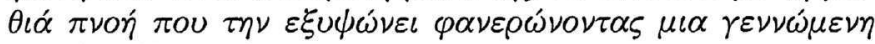
$\eta \theta \iota x \dot{\eta} \alpha \lambda \dot{\eta} \theta \varepsilon \iota \alpha) .{ }^{16}$

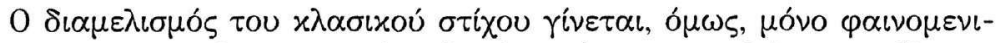

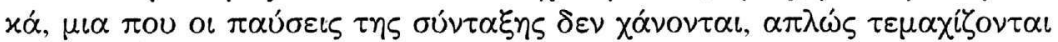

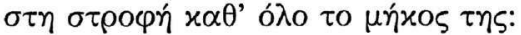

\begin{tabular}{|c|c|}
\hline$H \sigma \iota \omega \pi \dot{\eta} \pi \varepsilon \varepsilon^{\prime} \theta \varepsilon \iota$ & Ho sognato \\
\hline$\tau o \sigma \omega \mu \alpha$ & stanotte \\
\hline$H \gamma \alpha \dot{\lambda} \eta^{\prime} \nu \eta \iota \lambda \alpha^{\prime} \varepsilon \iota$ & una \\
\hline 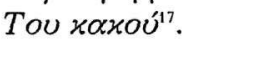 & $\begin{array}{l}\text { piana } \\
\text { striata }\end{array}$ \\
\hline$\Sigma \pi i \tau \iota \alpha \chi \tau i ́ \sigma \alpha \mu \varepsilon$ & d'una \\
\hline$\left.X \tau i \zeta{ }^{\prime}\right) \mu \varepsilon$ & freschezza \\
\hline 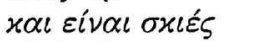 & \\
\hline$\alpha \delta \varepsilon \lambda \varphi \varepsilon \varepsilon^{\prime}$ & In veli \\
\hline 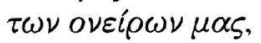 & varianti \\
\hline$\varepsilon v \alpha i \sigma \theta \eta \tau \varepsilon \varsigma$ & d'azzurr'oro \\
\hline$\sigma \alpha \nu \psi \nu \chi \varepsilon \varsigma^{18}$. & $\operatorname{alg} a^{19}$. \\
\hline
\end{tabular}




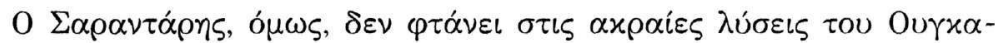

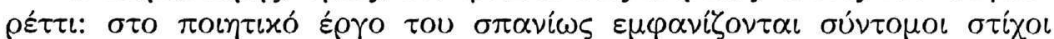

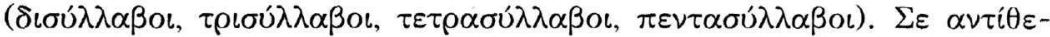

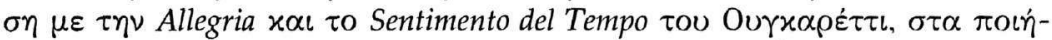

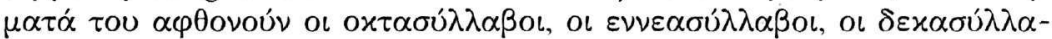

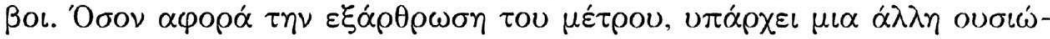

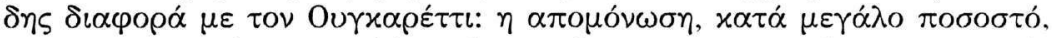

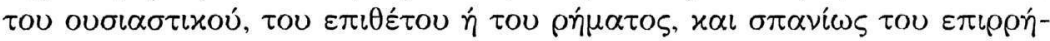

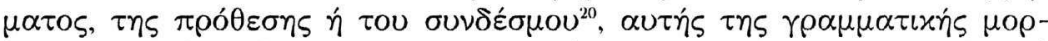

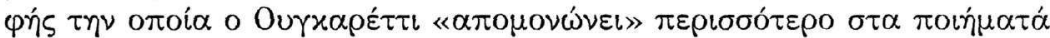

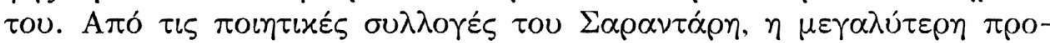

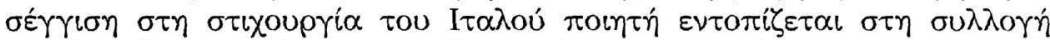

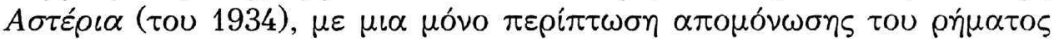

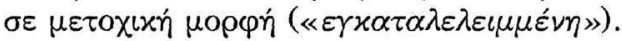

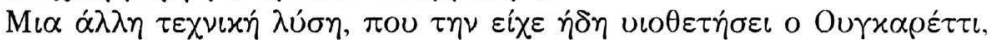

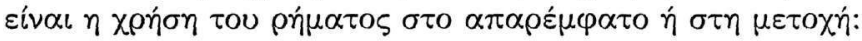

Ma vivere nell'io è angoscioso ${ }^{21}$
Ma non vivere di lamento come un cardellino accecato ${ }^{22}$

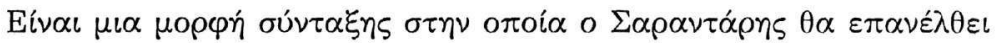

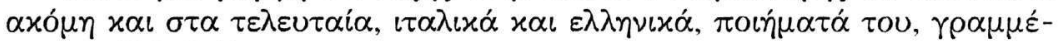

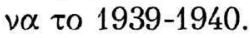

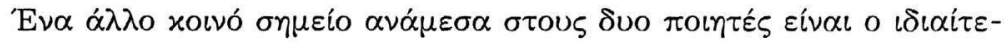

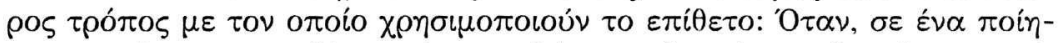

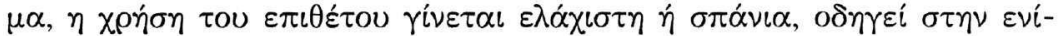

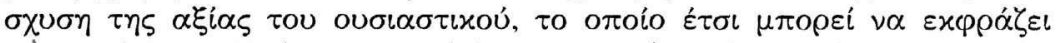

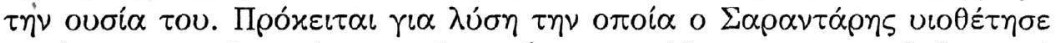

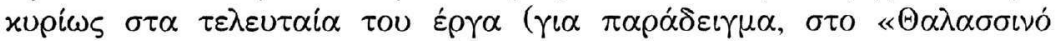

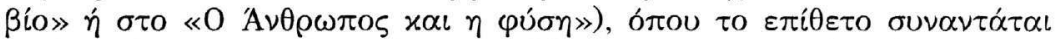

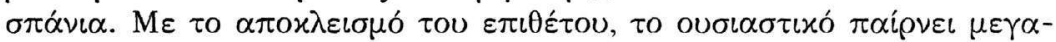

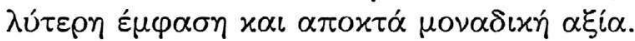

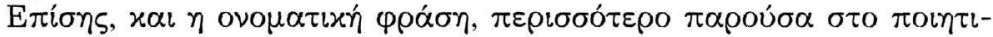

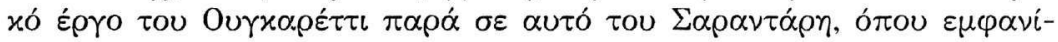

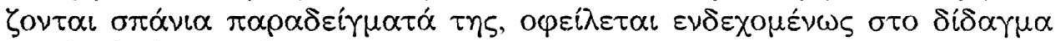

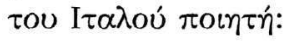

\author{
$\Phi u ́ \lambda \lambda \alpha \delta \varepsilon v \tau \rho O v$ \\ $\Phi \tau \varepsilon \rho \alpha ́ \alpha ~ \pi O U \lambda \iota o u ́$

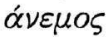 \\ $\varepsilon \dot{\tau} \tau \iota \tau \alpha \theta \alpha \dot{\lambda} \alpha \sigma \sigma \alpha$

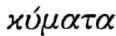




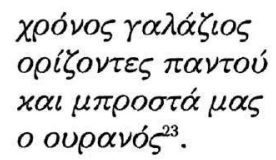

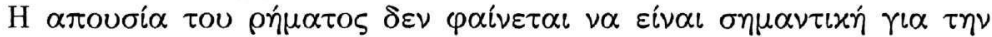

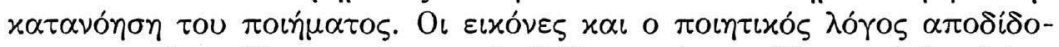

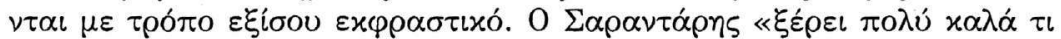

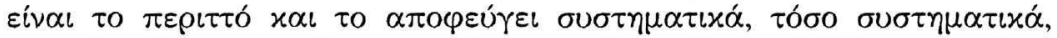

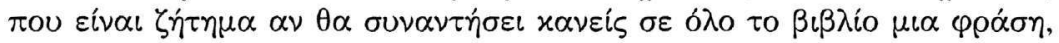

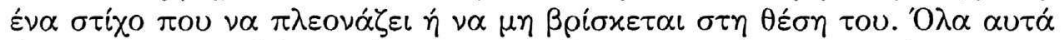

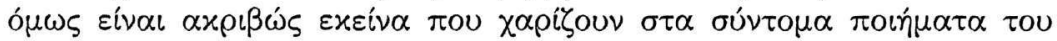

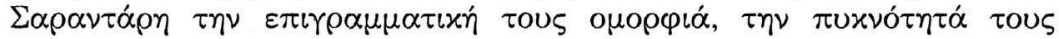

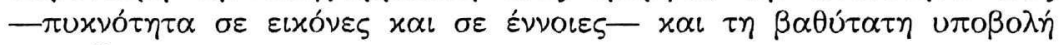

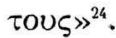

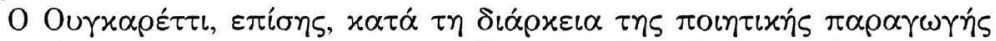

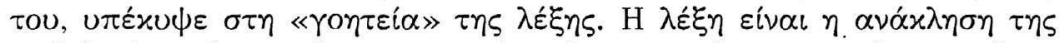

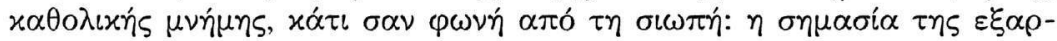

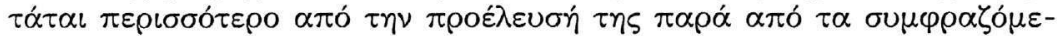

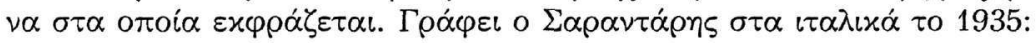

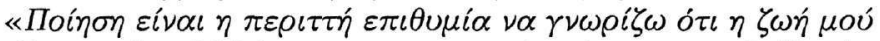

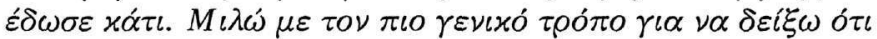

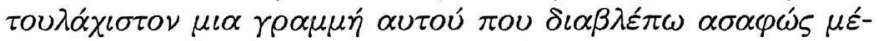

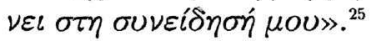

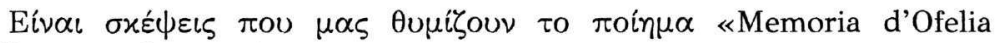

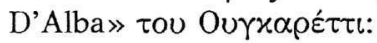

$E$ a fondo in breve del vostro silenzio

Si fermeranno

Cose consumate:

Emblemi eterni, nomi, Evocazioni pure...

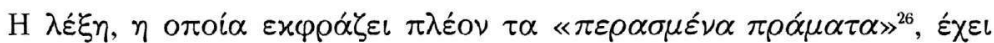

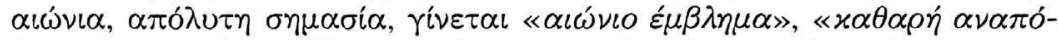

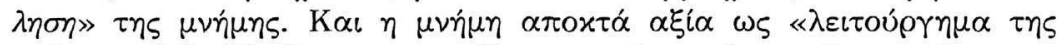

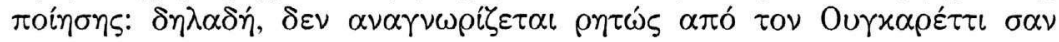

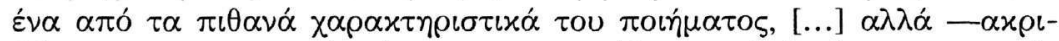

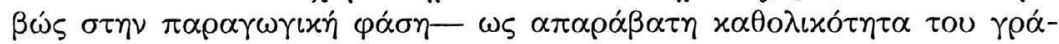

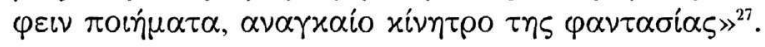




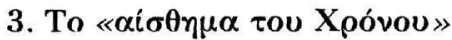

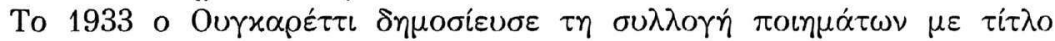

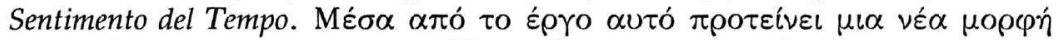

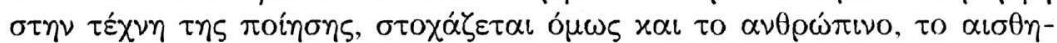

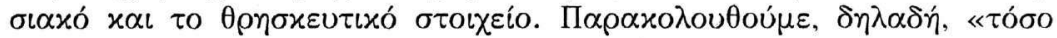

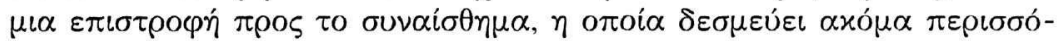

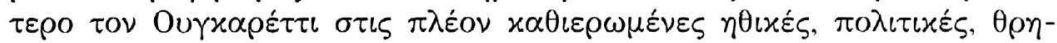

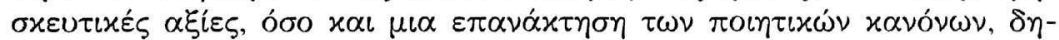

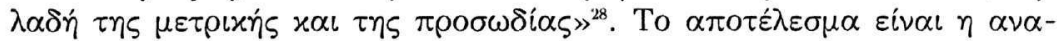

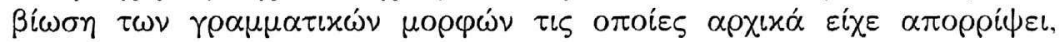

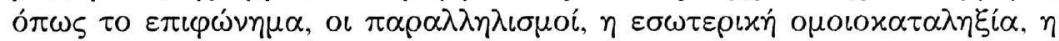

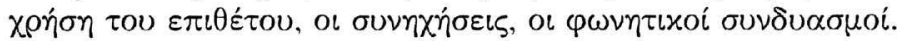

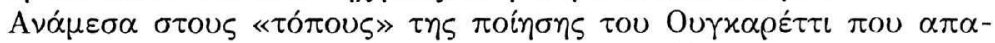

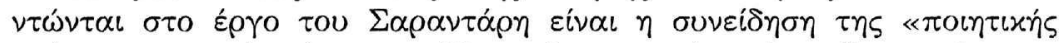

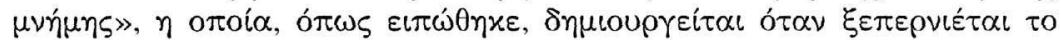

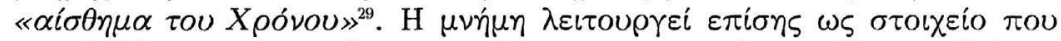

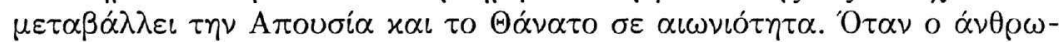

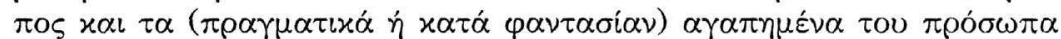

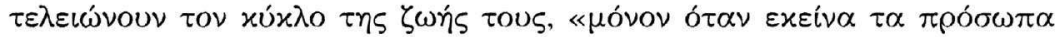

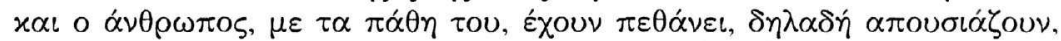

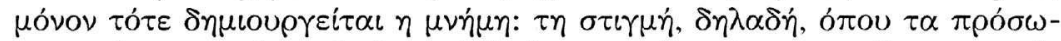

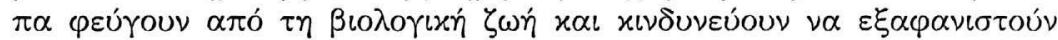

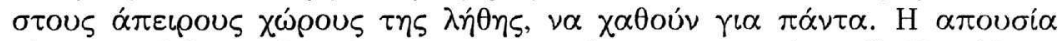

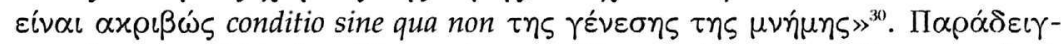

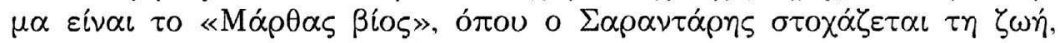

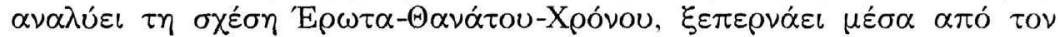

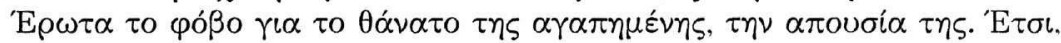

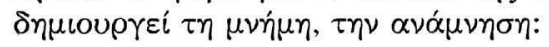

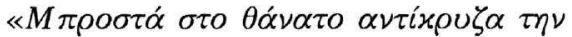

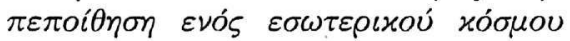

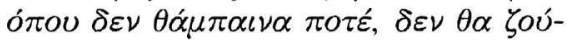

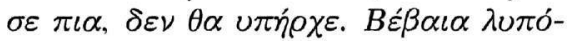

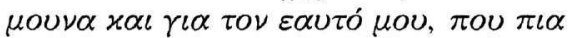

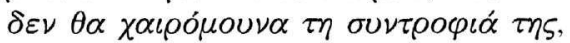

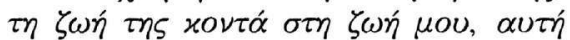

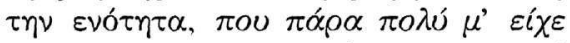

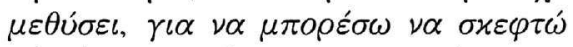

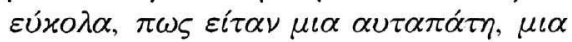

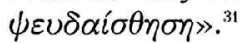

Amore, mio giovine emblema

Tornato a dorare la terra, Diffuso entro il giorno rupestre... Amore, salute lucente, Mi pesano gli anni venturi... Morte, arido fiume... Immemore sorella, morte, L'uguale mi farai del sogno Baciandomi $i^{32}$. 


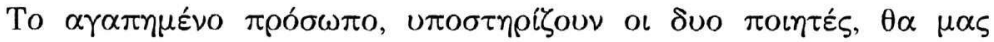

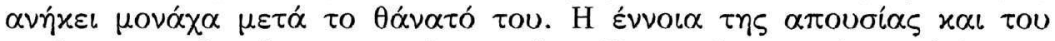

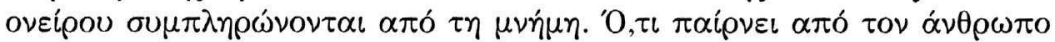

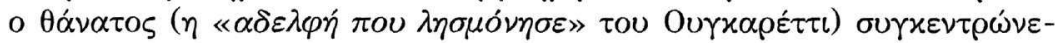

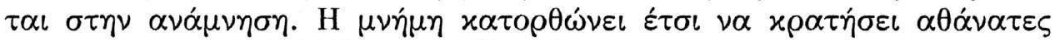

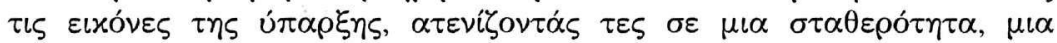

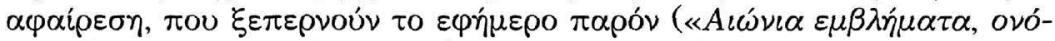

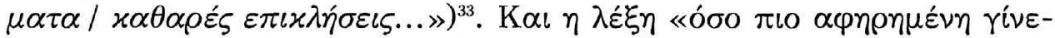

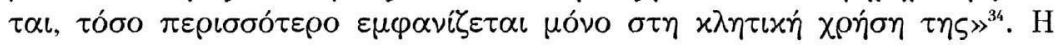

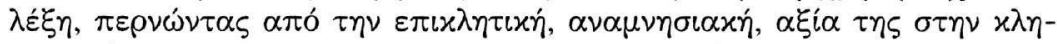

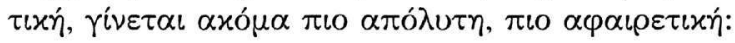
$X \alpha \dot{\nu \varepsilon \tau \alpha \iota ~ o ~ \chi \rho o ́ v o \varsigma, ~} \pi \alpha \iota \delta \iota \alpha^{35}$
$O$ gioventù
O uomini fratelli $i^{36}$
Passata è appena l'ora del distacco ${ }^{37}$
Di che reggimento siete, fratelli? $?^{38}$

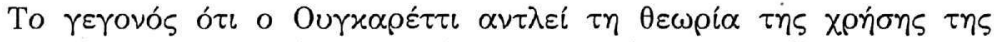

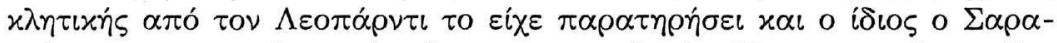

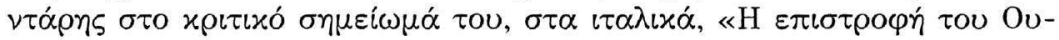
$\gamma$ $\gamma \propto \rho \varepsilon \tau \tau \iota \gg:$

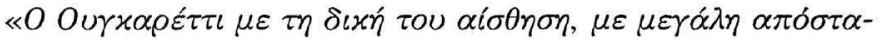

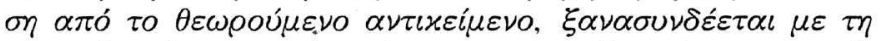

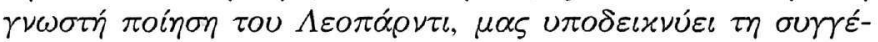

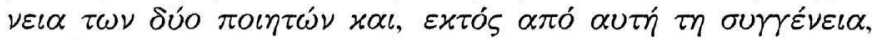

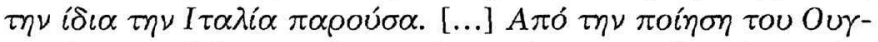

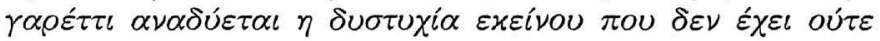

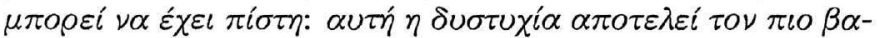

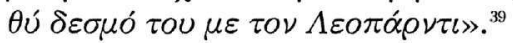

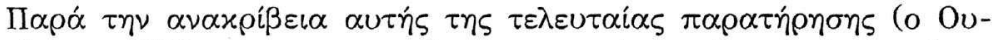

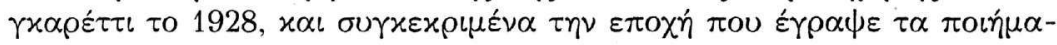

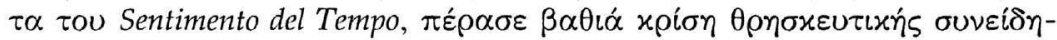

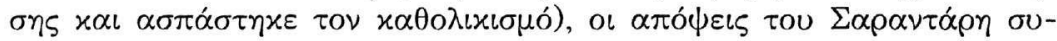

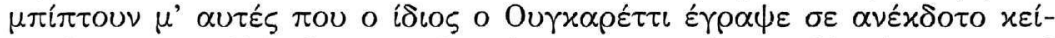

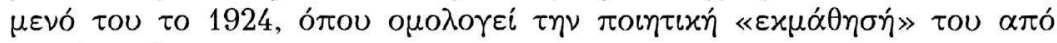

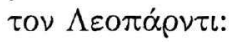

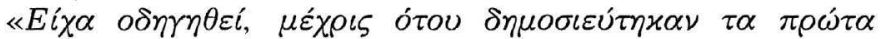

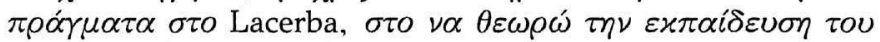

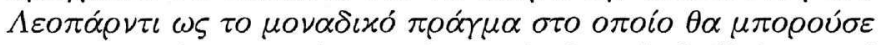

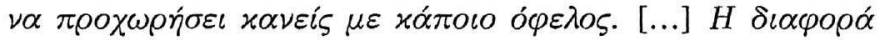

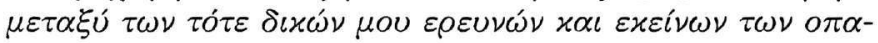

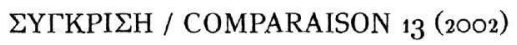




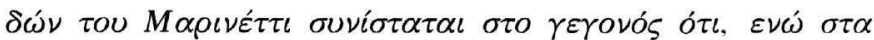

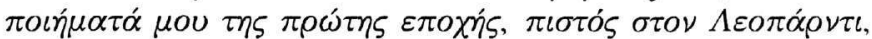

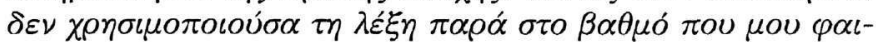

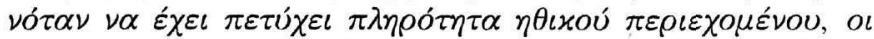

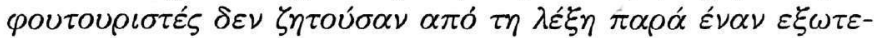

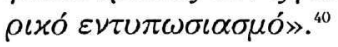

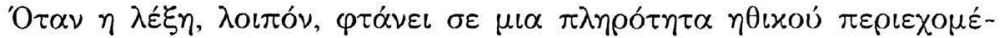

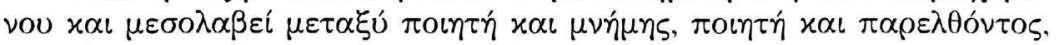

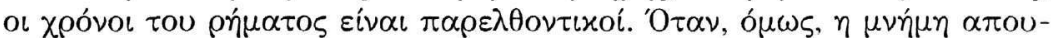

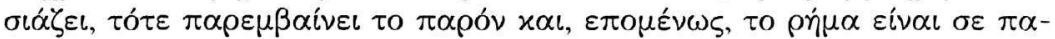

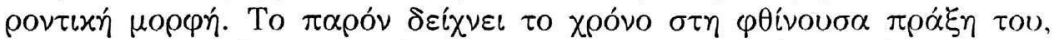

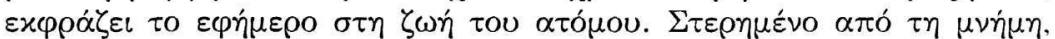

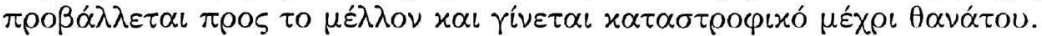

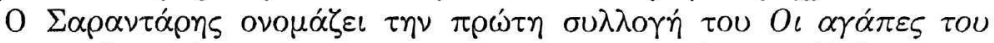

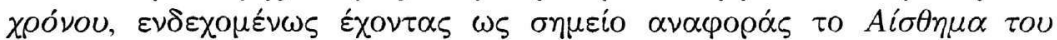

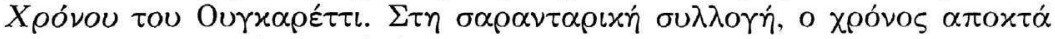

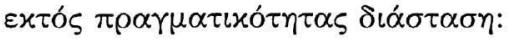

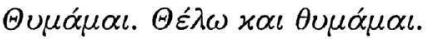 \\ $H \alpha \nu \alpha \dot{\mu} \nu \nu \eta \eta, \mu \varepsilon \tau \eta \nu \alpha \nu \alpha \tau \rho o \varphi \eta^{\prime}$,

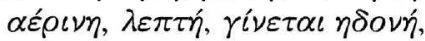

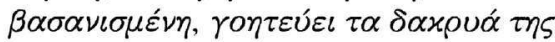 \\ $\mu \varepsilon \chi \alpha \mu o \gamma \varepsilon \dot{\lambda} \iota \alpha$. Eíve

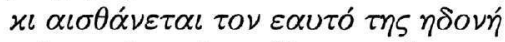 \\ $\alpha \varepsilon ́ \rho \iota \nu \eta, \lambda \varepsilon \pi \tau \eta^{\prime}, \alpha \nu \alpha ́ \lambda \alpha \varphi \rho \eta \ldots{ }^{41}$
}

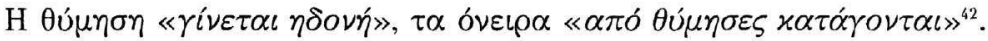

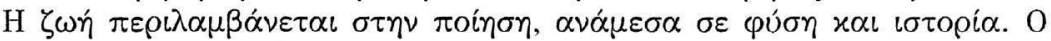

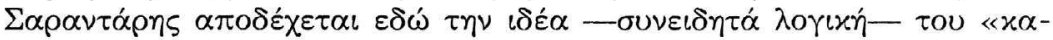
$\theta$ o

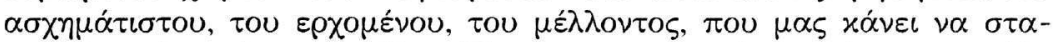

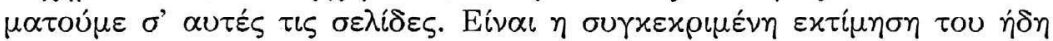

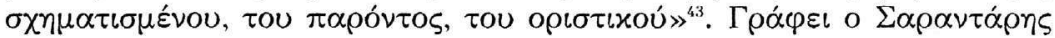

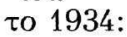

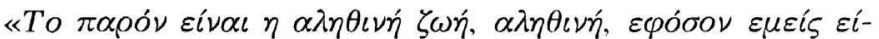

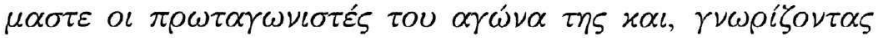

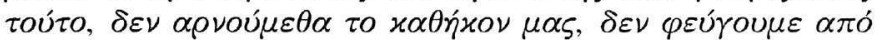

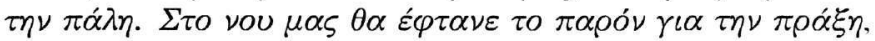

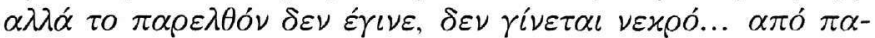

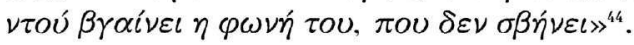




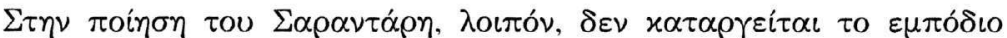

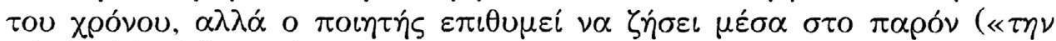

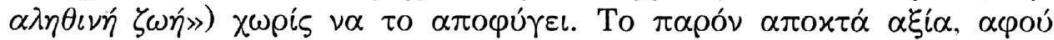

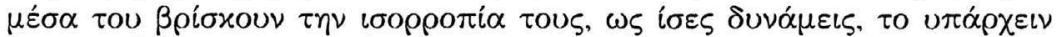

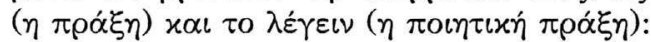

\begin{tabular}{|c|c|}
\hline 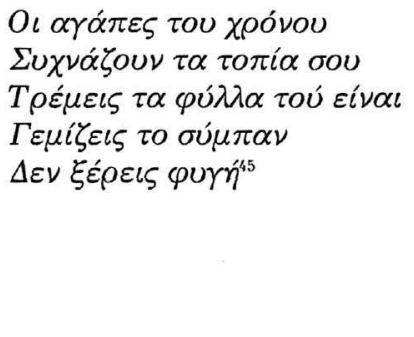 & $\begin{array}{l}\text { Ad ogni } \\
\text { clima } \\
\text { passato } \\
\text { mi trovo } \\
\text { languente } \\
\text { che una volta } \\
\text { gli ero } \\
\text { già stato } \\
\text { assuefatto }\end{array}$ \\
\hline
\end{tabular}

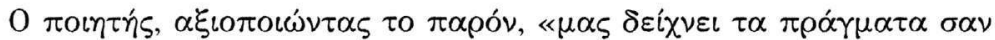

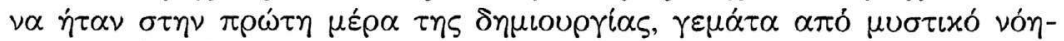

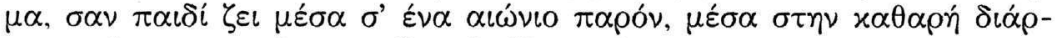

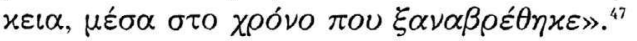

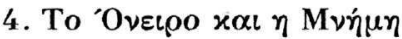

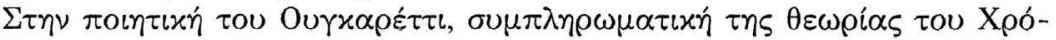

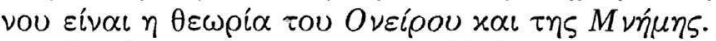

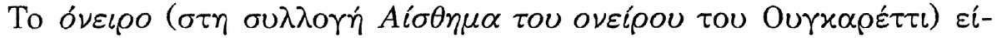

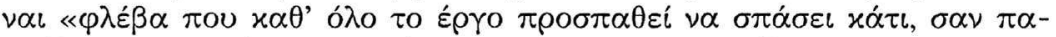

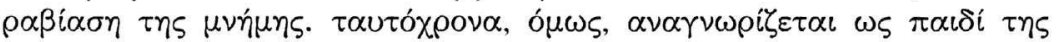

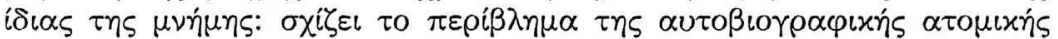

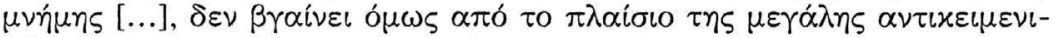

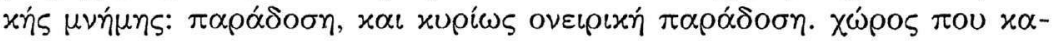

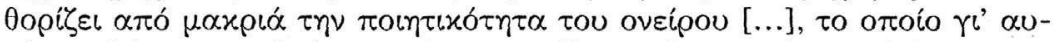

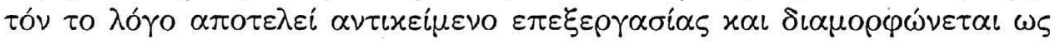

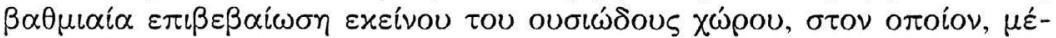

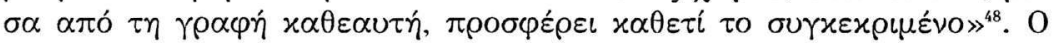

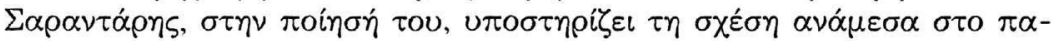

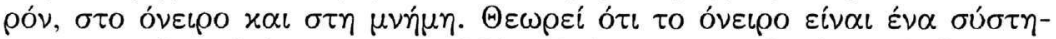

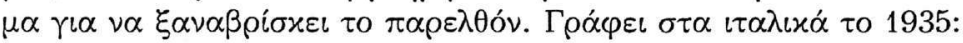

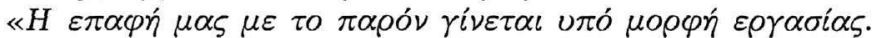

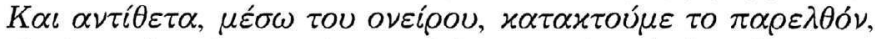

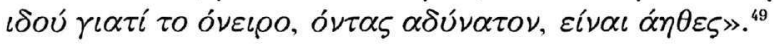

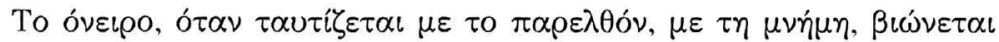




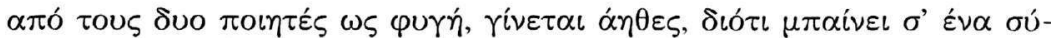

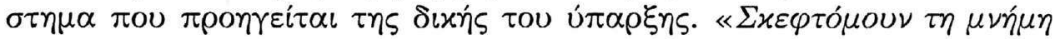

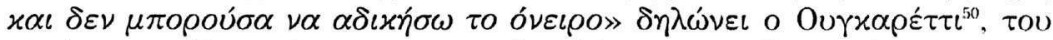

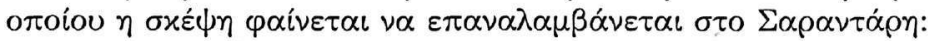

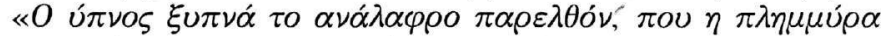

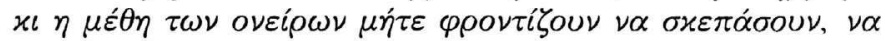

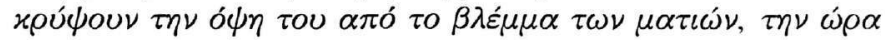

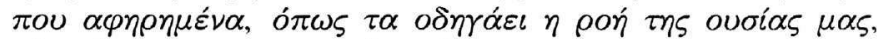

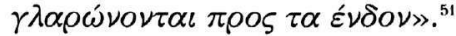

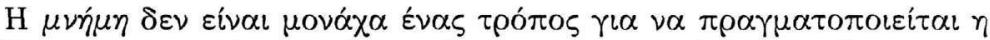

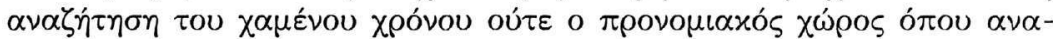

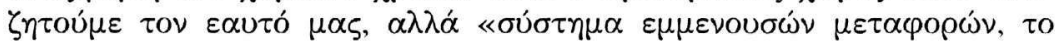

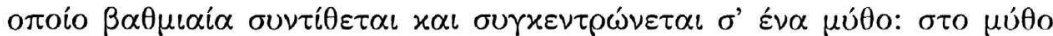

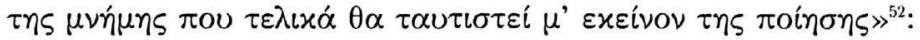

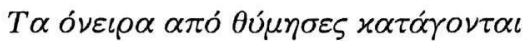

$O$ bel ricordo, siediti un momento ${ }^{54}$

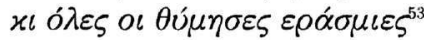

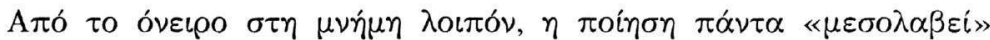

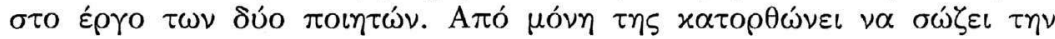

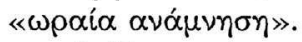

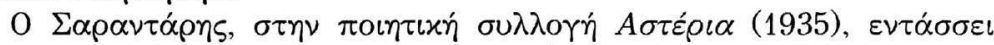

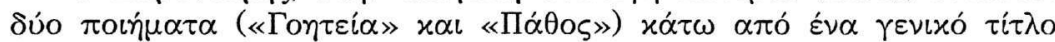

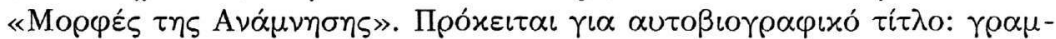

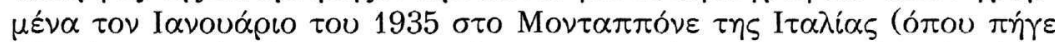

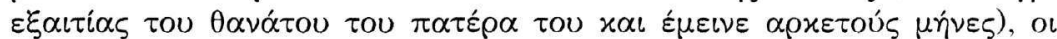

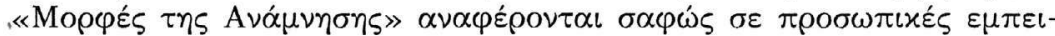

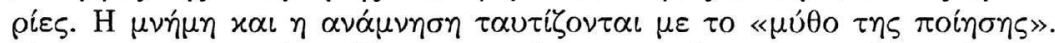

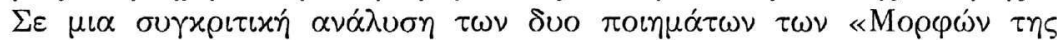

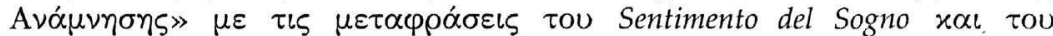

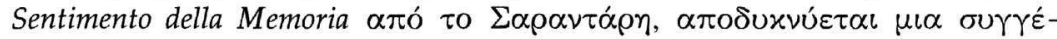

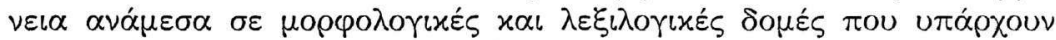

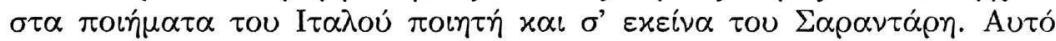

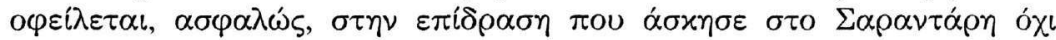

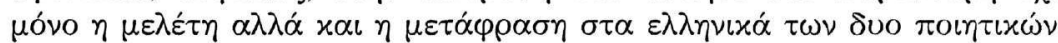

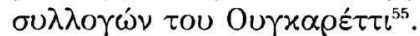

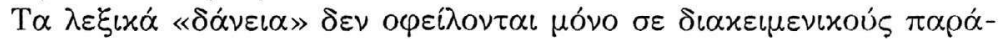

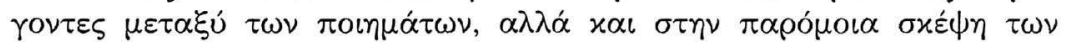

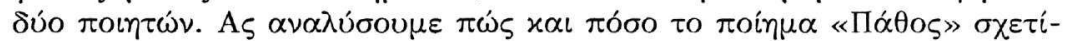

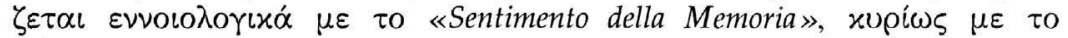
$\ll$ Canto terzo $»^{56}$ : 
$\Pi \mathrm{A} \Theta \mathrm{O \Sigma}$

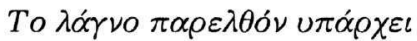

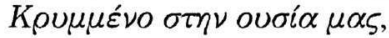

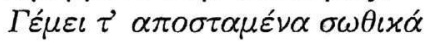

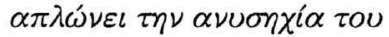

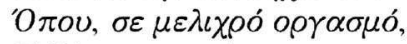

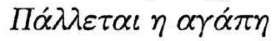

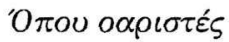

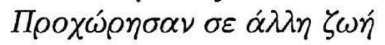

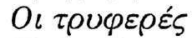

$\Pi \varepsilon \theta \alpha \mu \varepsilon \dot{v} \varepsilon \varsigma \eta \mu \varepsilon \rho \varepsilon$

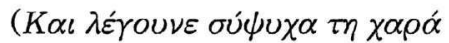
$\Sigma \tau^{\prime} \alpha o ́ \rho \alpha \tau \alpha$, $\Phi \rho o ́ v \iota \mu \alpha, \sigma \alpha \nu \mu v \sigma \tau \iota x o ́$

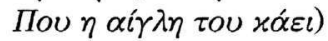

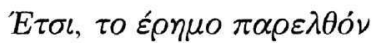

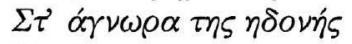

Broível

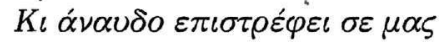

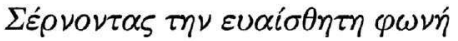
Tns ovoias

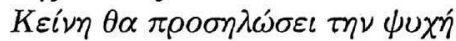

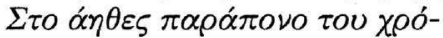
vov.

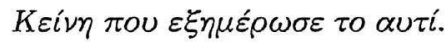

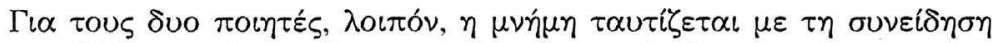

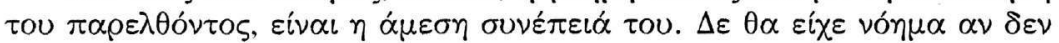

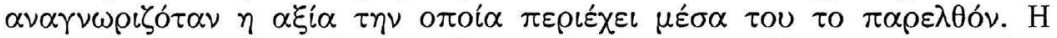

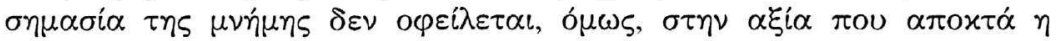

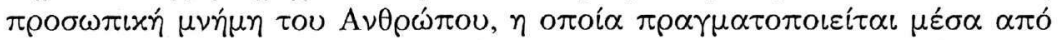

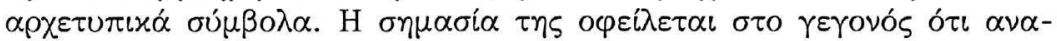

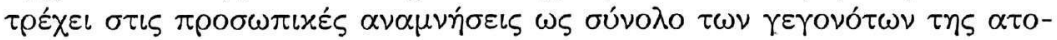

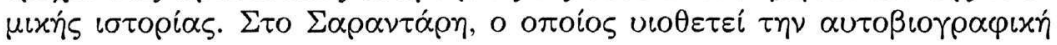

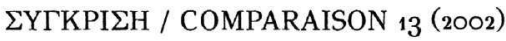

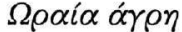

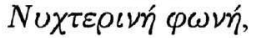

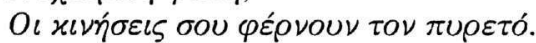

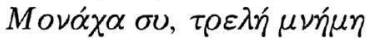

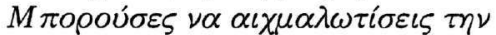
$\varepsilon \lambda \varepsilon v \theta \varepsilon \rho i \alpha$.

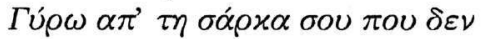
$\alpha \delta \rho \alpha ́ \chi \nu \varepsilon \tau \alpha \iota$,

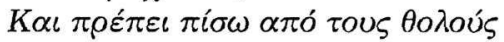
$\alpha \theta \rho \varepsilon ́ \varphi \tau \varepsilon \varsigma$,

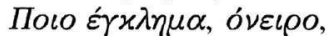

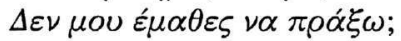

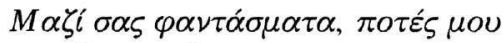
$\delta \varepsilon \nu \nu \tau \rho \varepsilon ́ \pi о \mu \alpha \ell$,

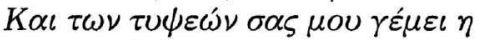
$\varkappa \propto \rho \delta \iota \alpha ́$.

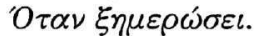




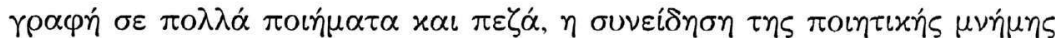

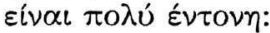

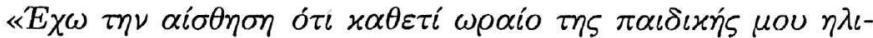

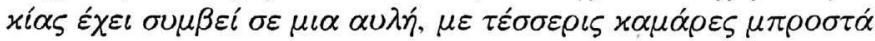

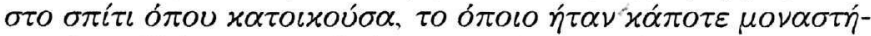

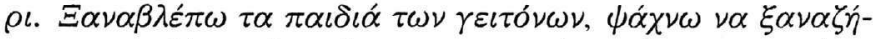

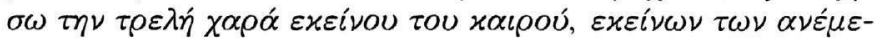

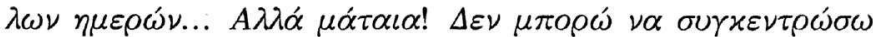

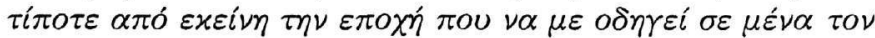

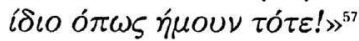

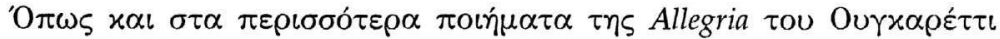

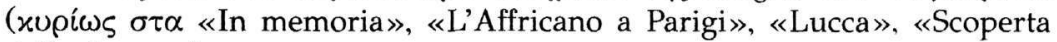
della donna»):

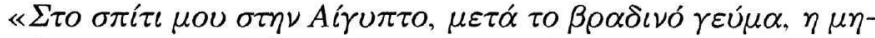

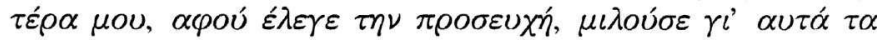

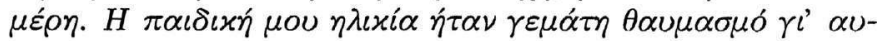
$\tau \alpha$.

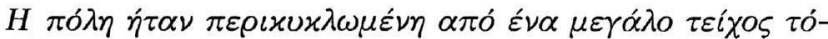

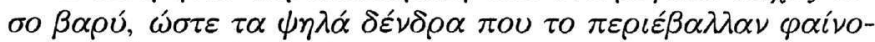
$\nu \tau \alpha \nu \alpha \varphi v ́ \sigma \iota x \alpha \varepsilon \lambda \alpha \varphi \rho \imath \alpha ́$.

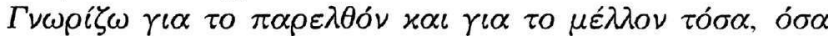

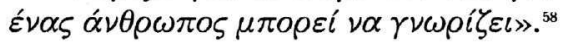

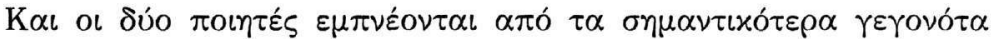

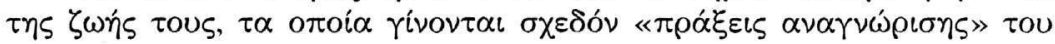

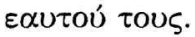

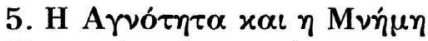

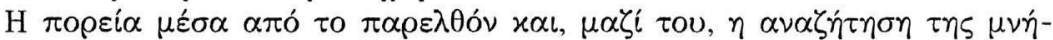

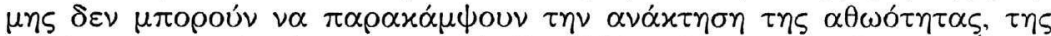

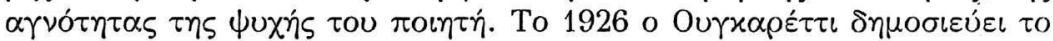
$\alpha ́ \alpha \theta \rho o$ «Innocenza e memoria» ${ }^{59}$, ó

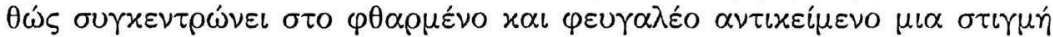

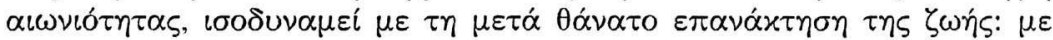

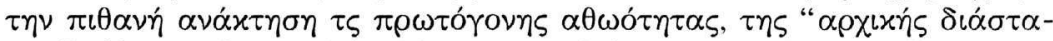

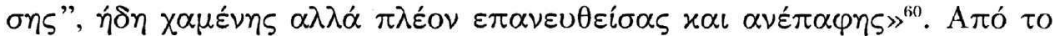

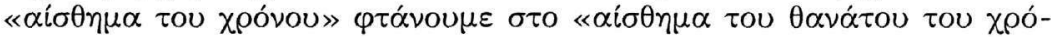

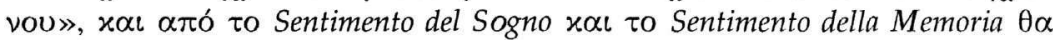

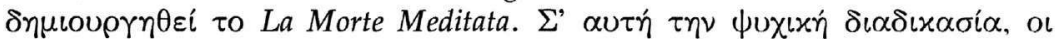

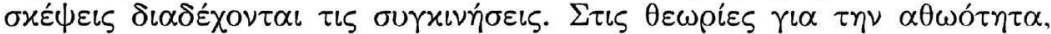

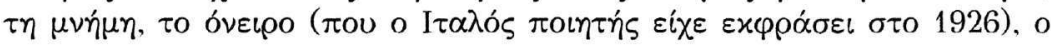




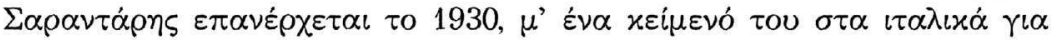

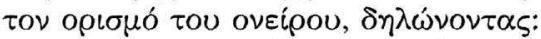

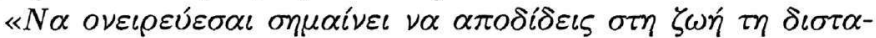

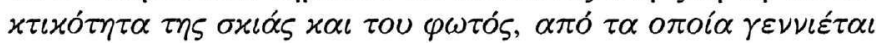

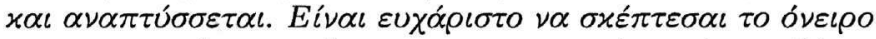

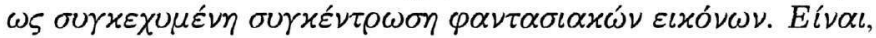

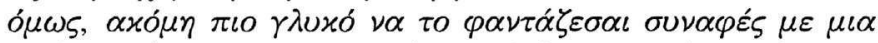

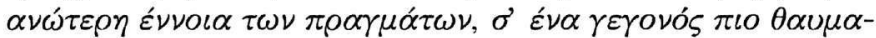

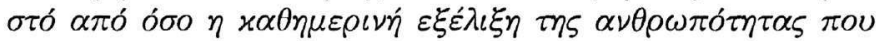
$\pi \rho \circ \chi \omega \rho \varepsilon i{ }^{61}$

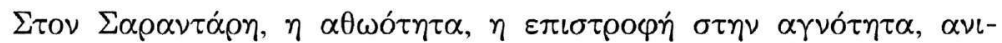

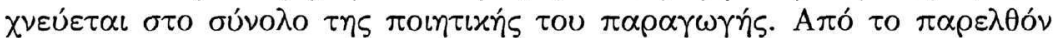

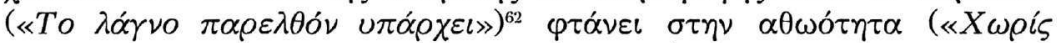

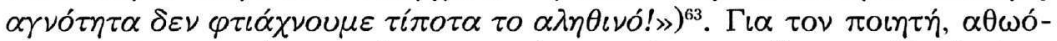

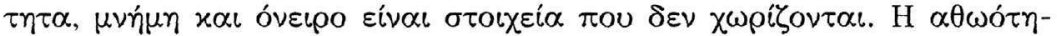

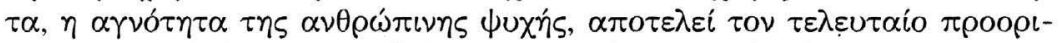

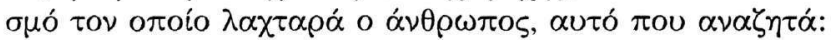

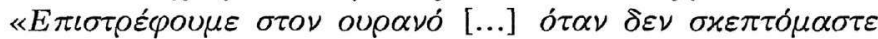

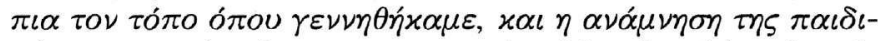

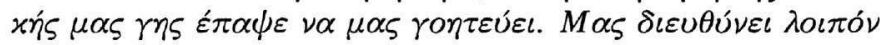

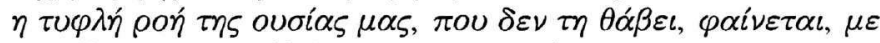

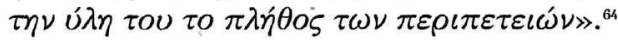

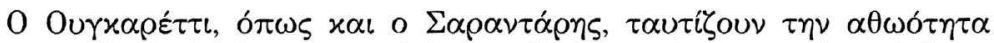

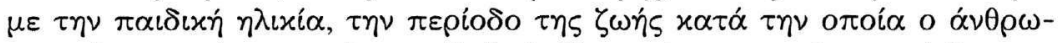

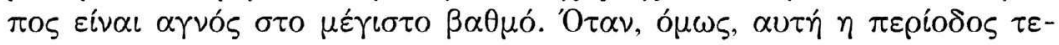

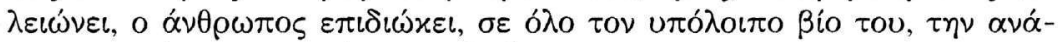

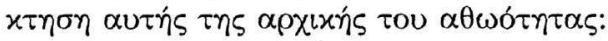

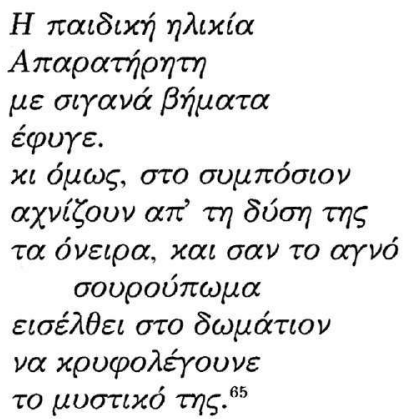

O gioventù

Passata è appena l'ora del distacco e già sono deserto

alti cieli della gioventù

libero slancio

età remota

perso in questa curva malinconia la notte sperde le lontananze stelle riapparsi nidi $d$ 'illusione. ${ }^{66}$ 


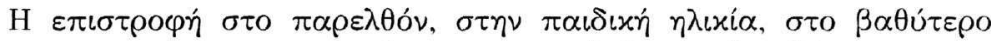

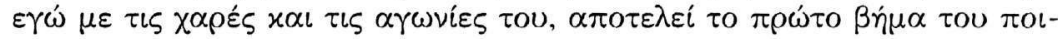

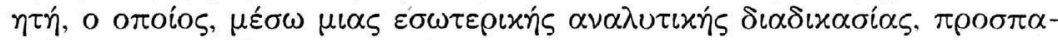

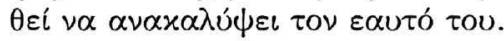

\section{6. $\mathrm{T} \alpha \alpha \rho \chi \varepsilon ́ \tau u \pi \alpha$}

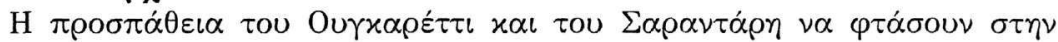

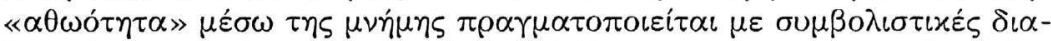

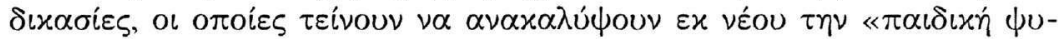

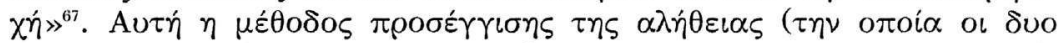

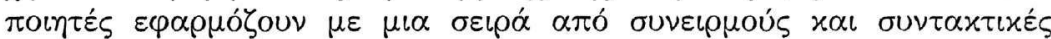

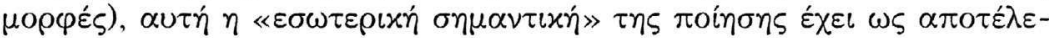

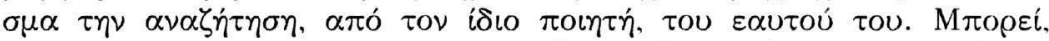

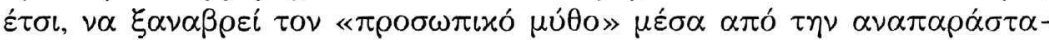

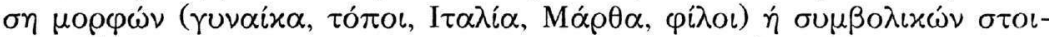

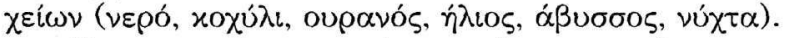

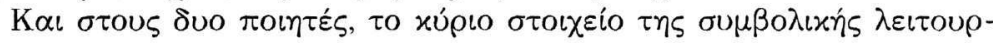

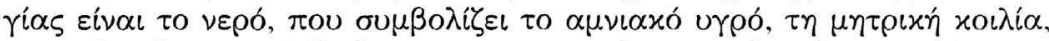

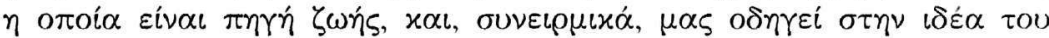

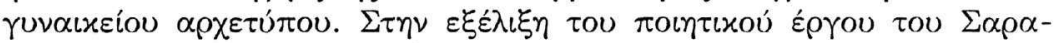

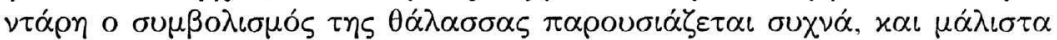

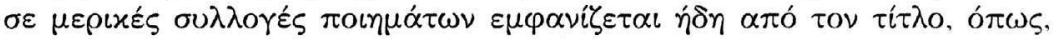

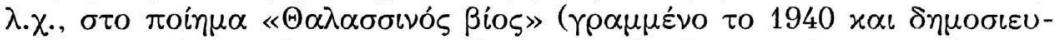

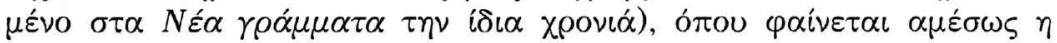

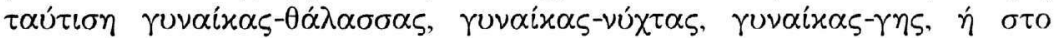

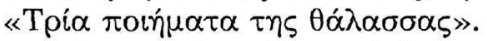

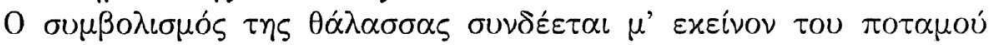

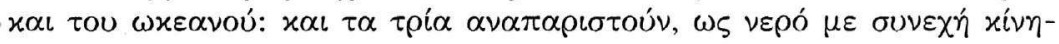

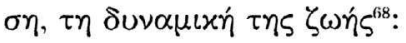

In quale mare

si confondono $i$ rivi del passato

$e$ dormono $i$ sogni dell'avvenire?

Il tempo non si raccoglie

$e$ il mare è introvabile ${ }^{69}$

\author{
Ho ripassato \\ le epoche \\ della mia vita \\ [...] questi sono i miei fiumi \\ contati nell'Isonzo ${ }^{70}$
}

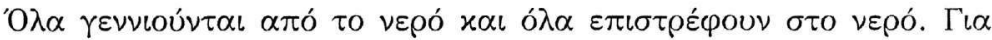

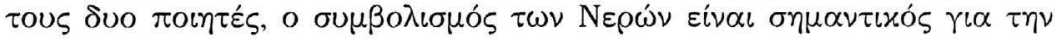

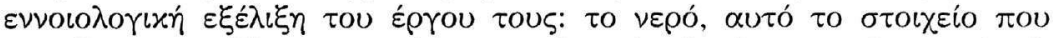

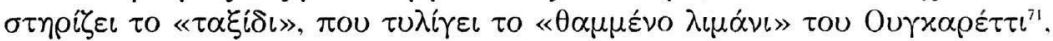

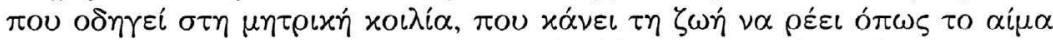

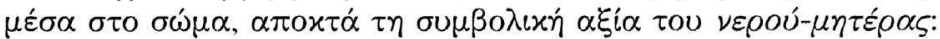




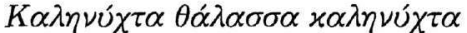

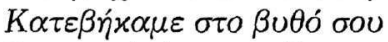

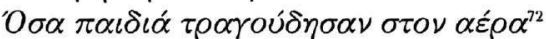

E subito riprende

il viaggio

come

dopo il naufragio

un superstite

lupo di mare ${ }^{73}$

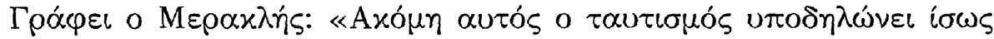

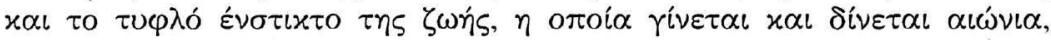

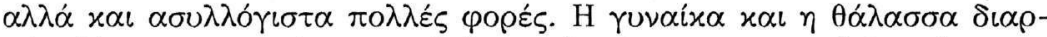

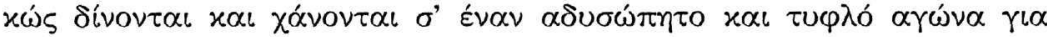

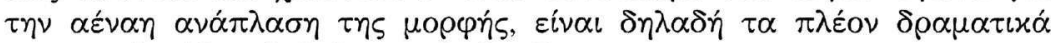

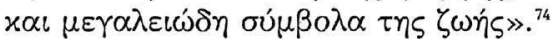

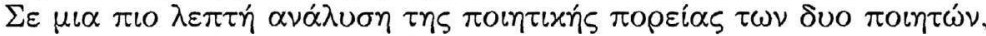

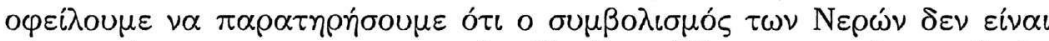

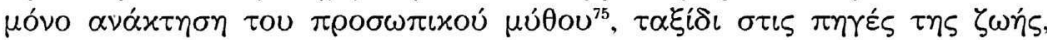

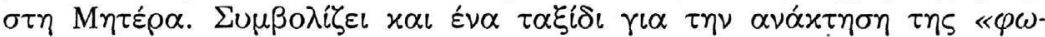

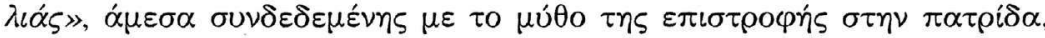

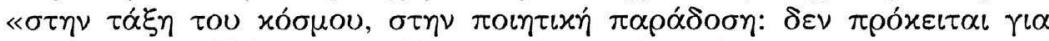

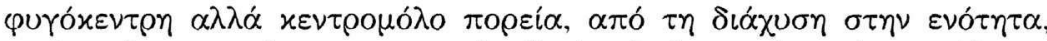

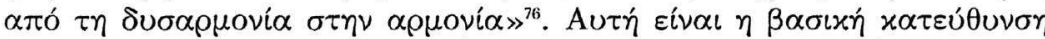

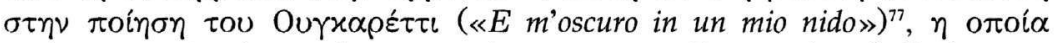

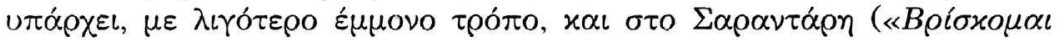
$\sigma \tau \eta \varphi \omega \lambda \iota \alpha \dot{\alpha} \mu \nu »)^{78}$.

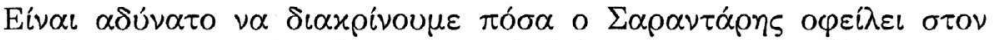

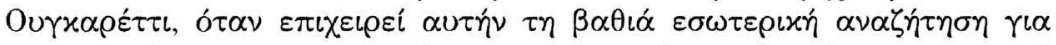

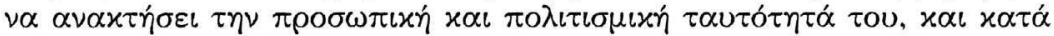

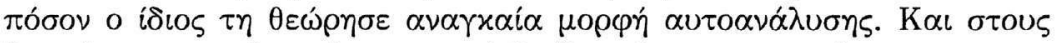

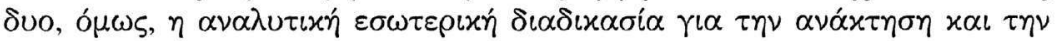

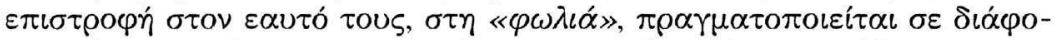

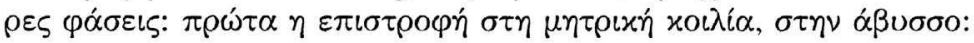

$K \alpha \lambda \eta \nu u ́ \chi \tau \alpha, \theta \alpha \dot{\lambda} \alpha \sigma \sigma \alpha, \varkappa \alpha \lambda \eta \nu u ́ \chi \tau \alpha$

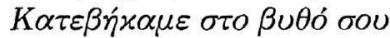

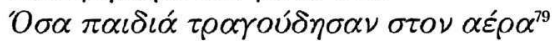

\section{Col mare}

mi sono fatto

una bara

di freschezza $a^{80}$

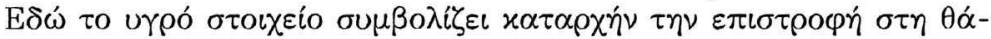

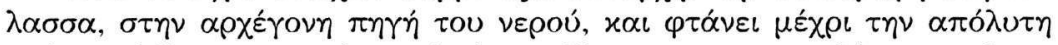

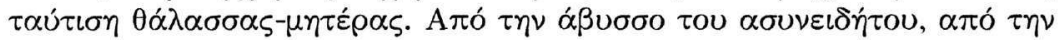

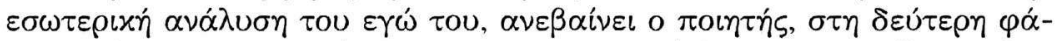
$\sigma \eta, \varepsilon \pi \alpha \dot{\alpha} \nu \omega \sigma \tau \eta \eta, \delta \eta \lambda \alpha \delta \tilde{\eta} \sigma \tau \eta \nu \pi \rho \alpha \gamma \mu \alpha \tau \iota x \dot{\eta} \zeta \omega \dot{\eta}, \gamma \iota \alpha \nu \alpha \xi \alpha \nu \alpha \rho \chi i \sigma \varepsilon l$

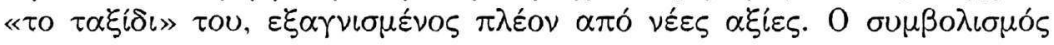




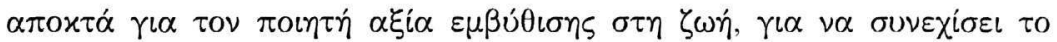

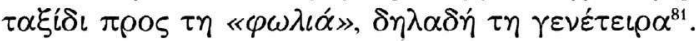

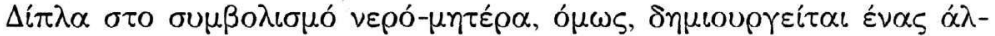

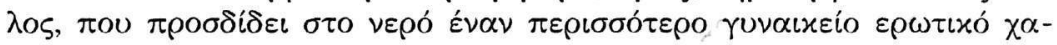

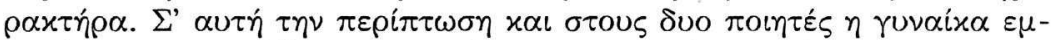

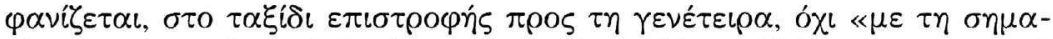

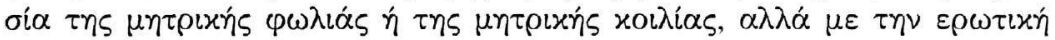

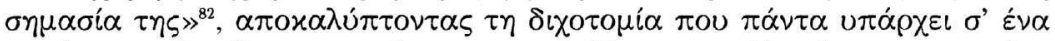

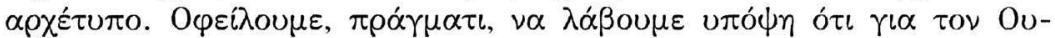

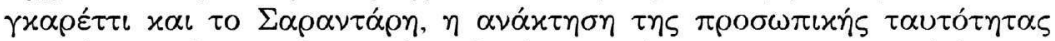

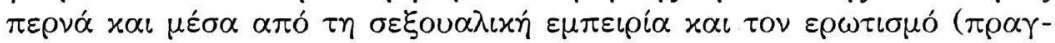

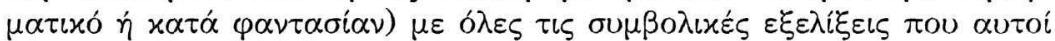

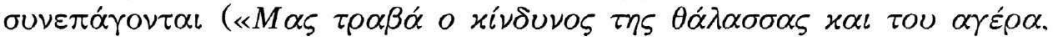

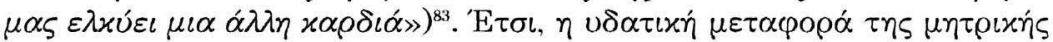

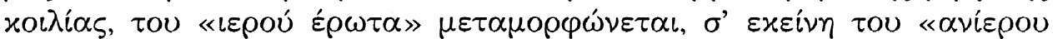

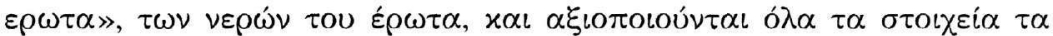

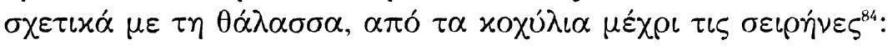

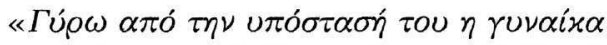

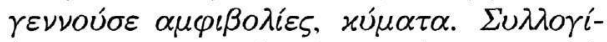

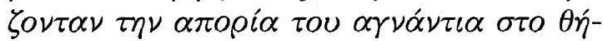

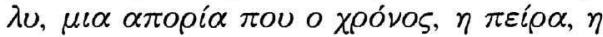

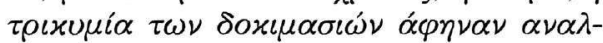

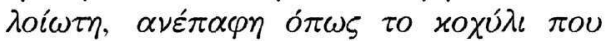

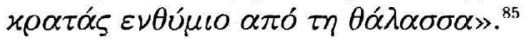

Al buio campestre fragranti svolazzi di maree raccordati nelle conchiglie amuleto d'amore ${ }^{86}$.

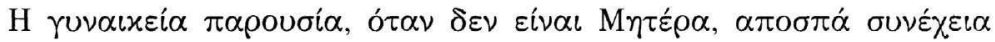

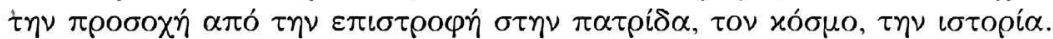

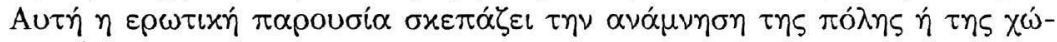

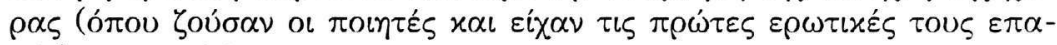

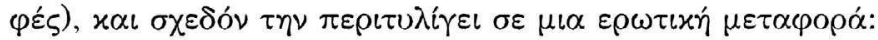

$\Omega I \tau \alpha \lambda i \alpha, \pi o \iota \alpha \theta \dot{\alpha} \lambda \alpha \sigma \sigma \alpha \pi \varepsilon \theta \alpha i v \varepsilon \iota$,

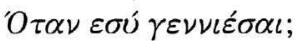

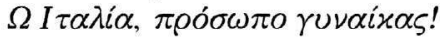

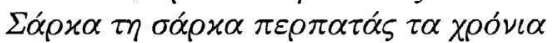

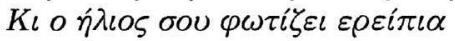

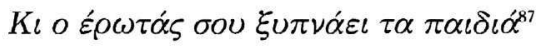

Ho visto

la mia città sparire

lasciando

un poco

un abbraccio di lumi nell 'aria torbida

sospesi $i^{88}$

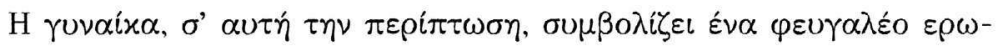




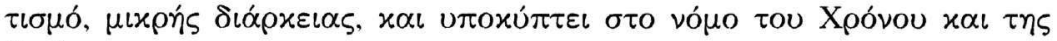
Zw'́s.

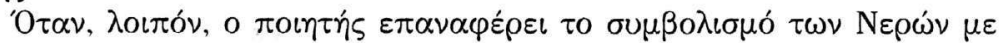

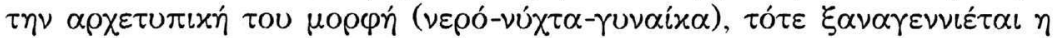

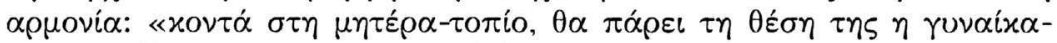

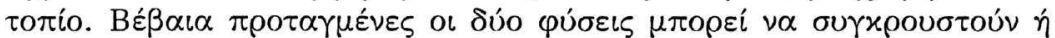

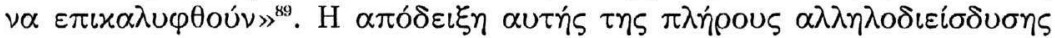

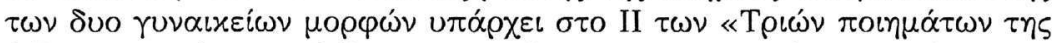

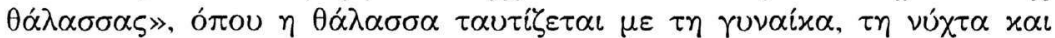

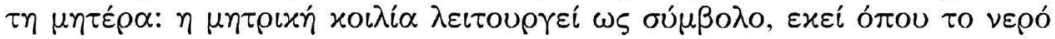

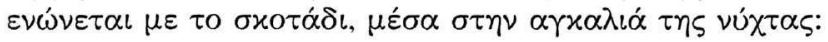

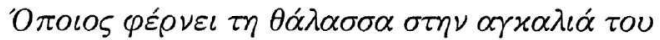

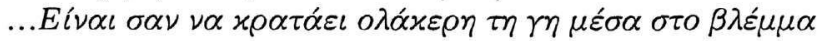

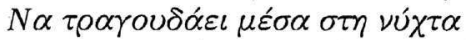

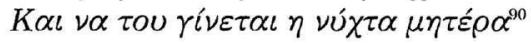

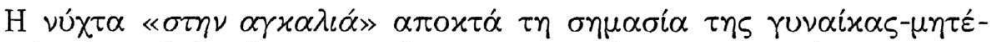

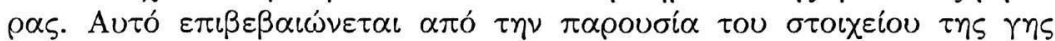

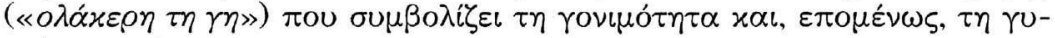

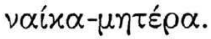

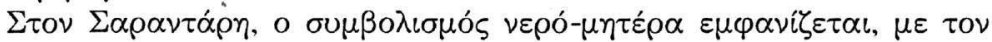

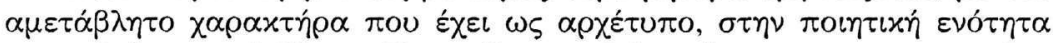

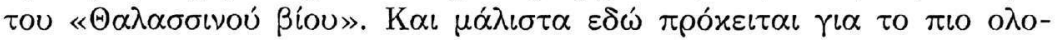

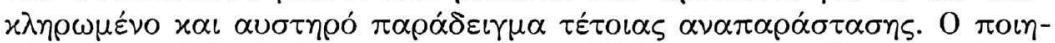

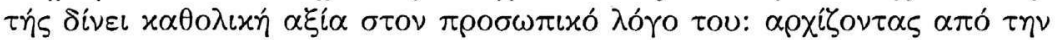

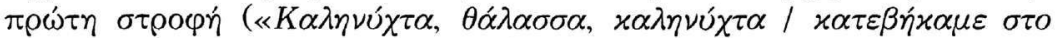
$\beta \nu \theta o ́$ бov»), ó

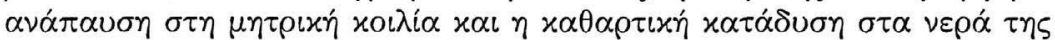

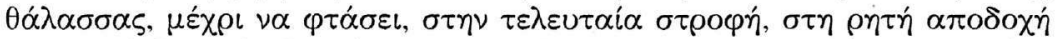

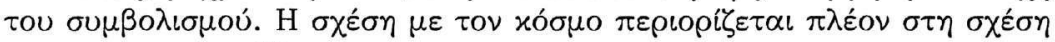

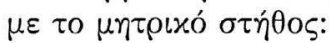

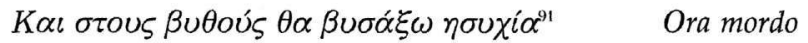
come un neonato la mammella lo spazio ${ }^{92}$

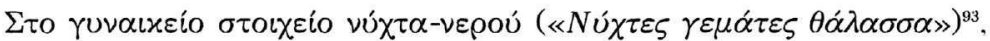

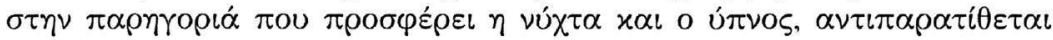

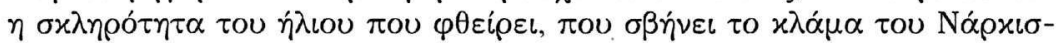

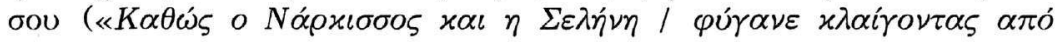




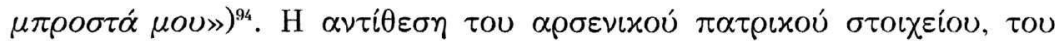

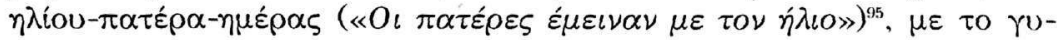

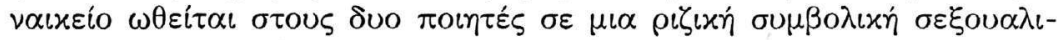

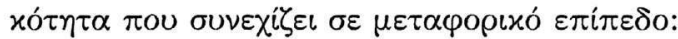

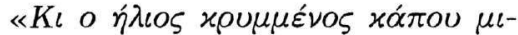
$\lambda \alpha \dot{\varepsilon} \varepsilon l \mu \varepsilon \tau \eta \nu \nu u ́ \chi \tau \alpha \varepsilon x \varepsilon i \nu \eta, \alpha \pi$ 'ó $\pi o v$

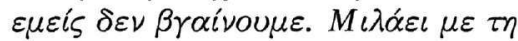

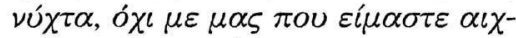

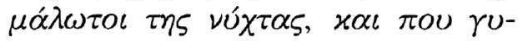

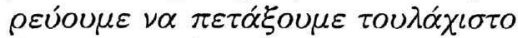

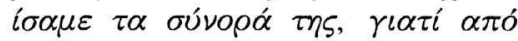

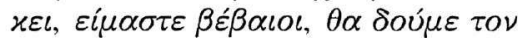
$\dot{\eta} \lambda \iota 0 » .^{96}$
Il sole si semina in diamanti di gocciole d'acqua sull'erba flessuosa ${ }^{97}$

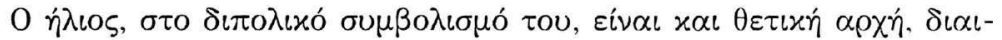

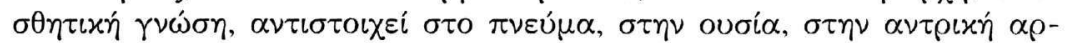
$\chi \dot{\eta}$.

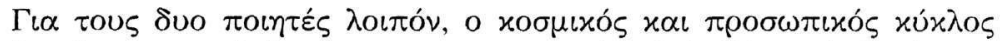

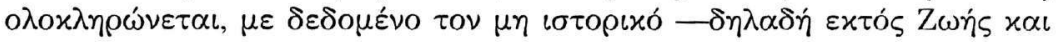

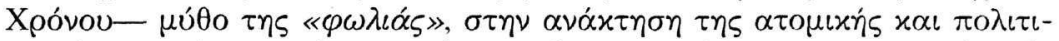

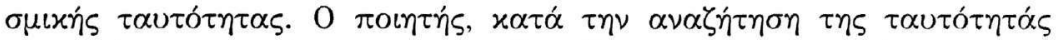

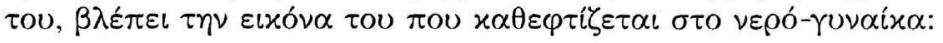

$\Theta \dot{\alpha} \lambda \alpha \sigma \sigma \alpha, i \sigma \omega \varsigma \theta \dot{u} \mu \alpha$

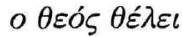

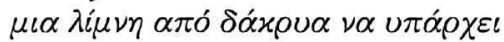

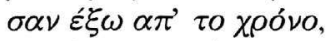

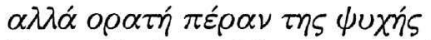

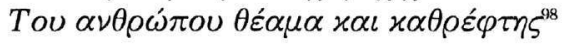

\author{
E l'uomo \\ curvato \\ sull'acqua \\ sorpresa dal sole \\ si rinviene \\ un'ombra
}

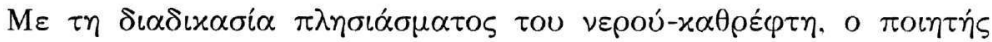

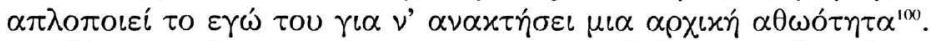

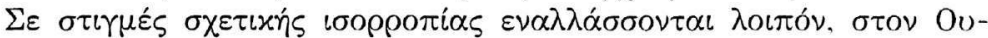

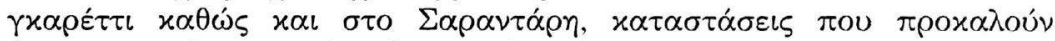

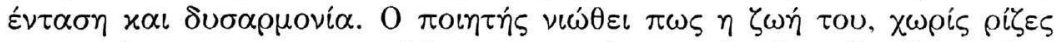

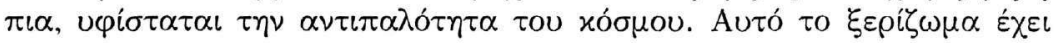




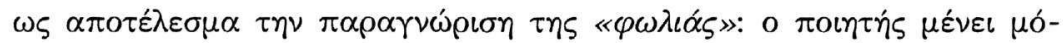

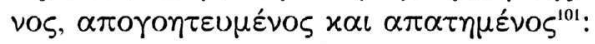

\begin{tabular}{|c|c|}
\hline 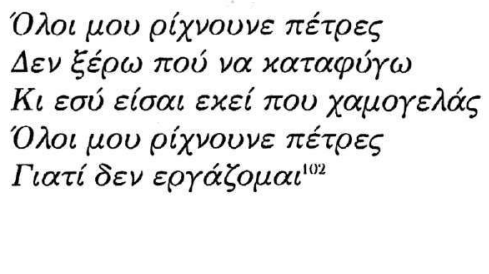 & $\begin{array}{l}\text { In un } \\
\text { infinito } \\
\text { che mi calca e mi spreme } \\
\text { col suo } \\
\text { fievole } \\
\text { tatto } \\
\text { fluente }^{103}\end{array}$ \\
\hline
\end{tabular}

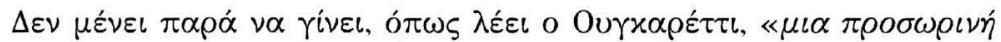

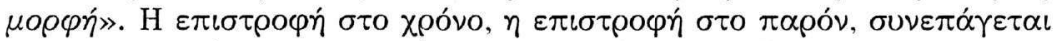

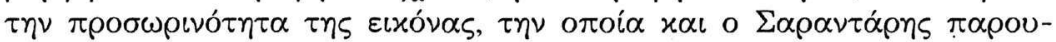

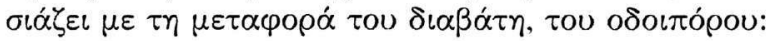

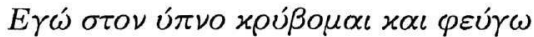

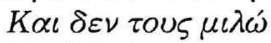

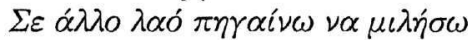

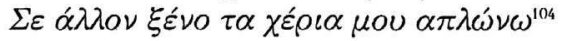

Mi riconosco

immagine

passeggera

Presa in un giro immortale ${ }^{105}$

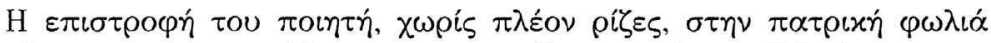

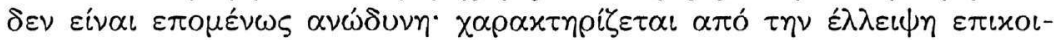

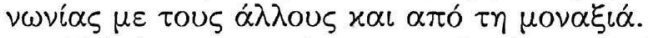

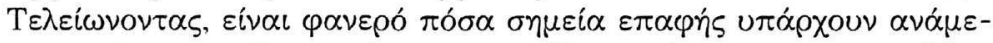

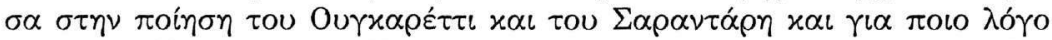

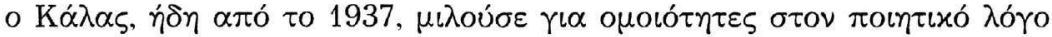

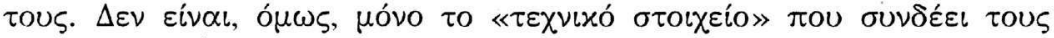

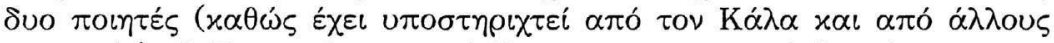

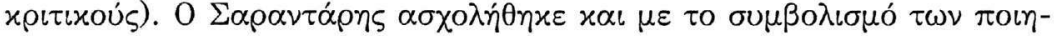

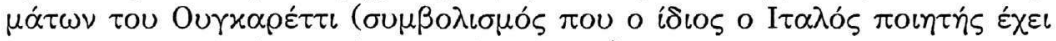

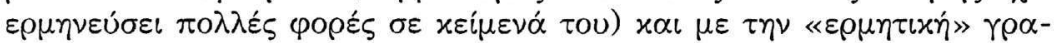

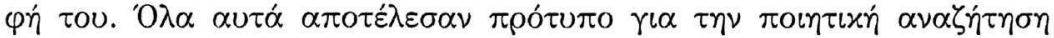

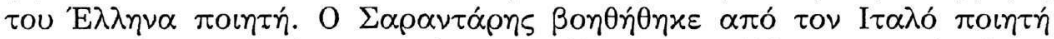

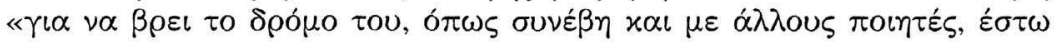

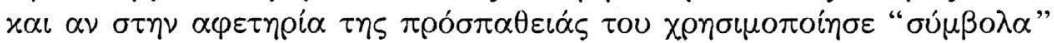

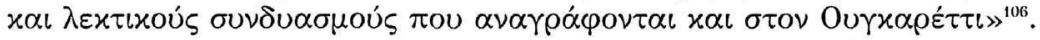




\section{$\Sigma \eta \mu \varepsilon \iota \omega ́ \sigma \varepsilon \iota \zeta$}

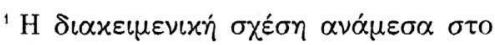

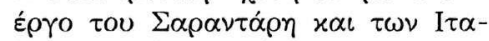

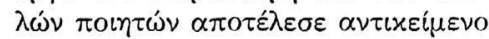

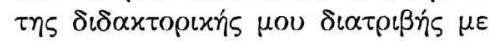

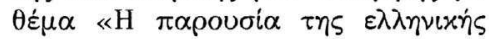

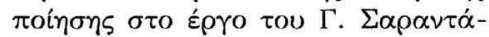

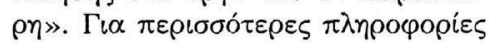

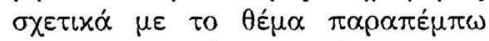

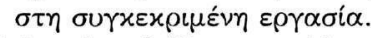

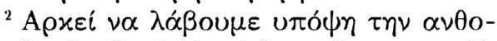

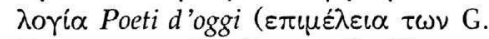
Papini $x \propto \iota$ P. Pancrazi) Firenze.

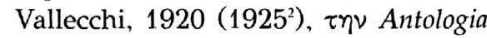
della Diana, Napoli, Libreria della Diana, 1918.

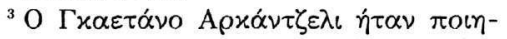

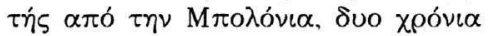

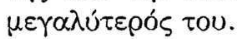

${ }^{4}$ F.M. Pontani, Inediti italiani di Sarandaris, Roma, Italograeca, 1965. $\sigma \varepsilon \lambda .34$.

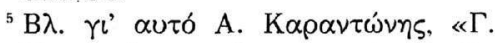
$\Sigma \alpha \rho \alpha \nu \tau \alpha ́ \alpha \eta \varsigma », \Phi v \sigma \iota \gamma \nu \omega \mu i \varepsilon \zeta, \tau$. B',

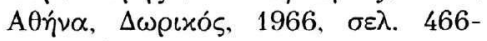
467, 472.

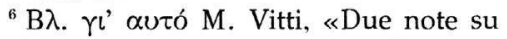
Ungaretti in Grecia», $\pi \varepsilon \rho$. Prospetti,

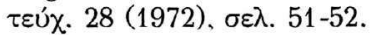

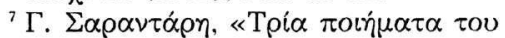

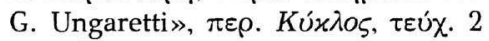

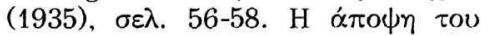

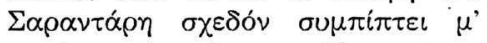

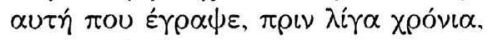

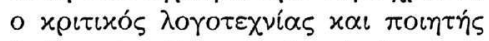

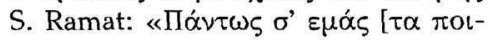

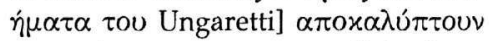

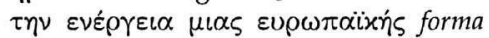

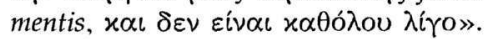
(S. Ramat, «Sentimento del tempo di Giuseppe Ungaretti», $\pi \varepsilon \rho$. Poesia,

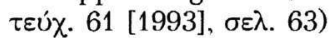

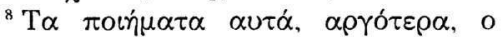

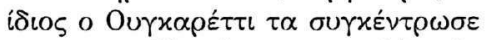

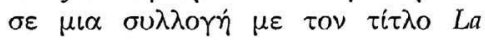

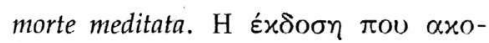

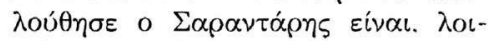

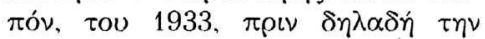

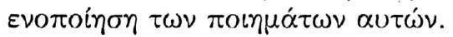

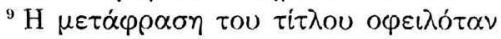

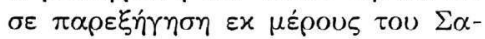

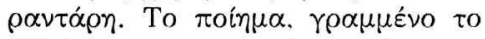

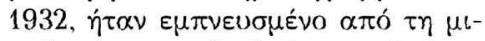

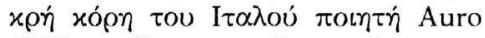

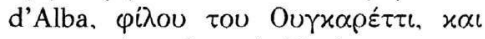

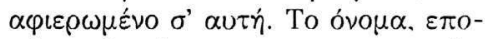

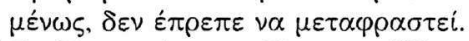

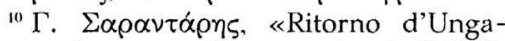

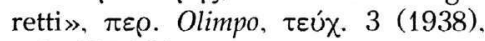
$\sigma \varepsilon \lambda .221-224$.

" G. Ungaretti, «Ragioni d'una poesia». Vita d'un tomo. Tutte le poesie, Milano. Mondadori, 1982, $\sigma \varepsilon \lambda$. L XXVI.

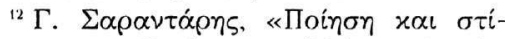

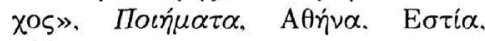
1961, $\sigma \varepsilon \lambda .4$.

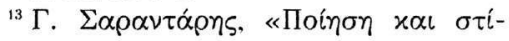

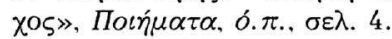

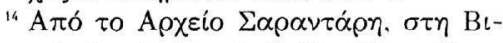

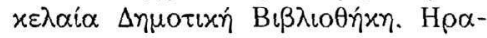

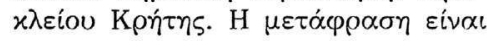

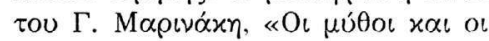

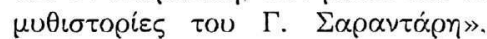

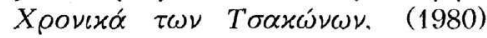

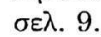

${ }^{15} \mathrm{G}$. Ungaretti, «Ragioni d'una poesia». Vita d'un uomo. Tutte le poesie. ó. $\pi$. $\sigma \varepsilon \lambda$. L XXVI-L XXVII.

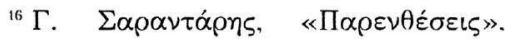

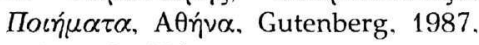

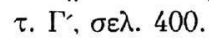

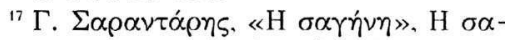

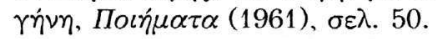

${ }^{18} \Gamma$. $\Sigma \alpha \rho \alpha \nu \tau \dot{\rho} \rho \eta \varsigma, ~ « \Sigma \pi i \tau \iota \alpha \chi \tau i \sigma \alpha \mu \varepsilon \ldots »$.

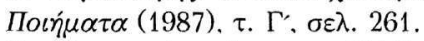

${ }^{19} \mathrm{G}$. Ungaretti, «Sogno», L'Allegria. Vita d'un uomo. Tutte le poesie. ó. $\pi$. $\sigma \varepsilon \lambda .76$.

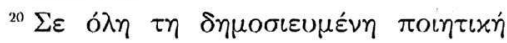




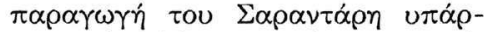

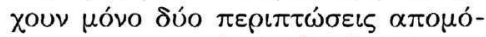

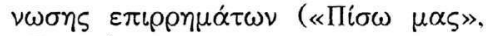
«Tóte»).

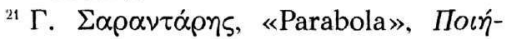

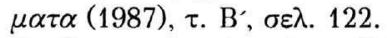

${ }^{22} \mathrm{G}$. Ungaretti, «Agonia», L'Allegria, Vita d'un uomo. Tutte le poesie, ó. $\pi$., $\sigma \varepsilon \lambda .10$.

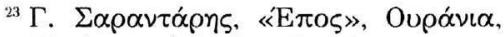

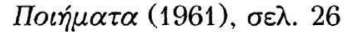

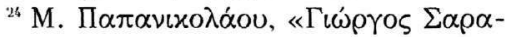

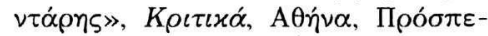

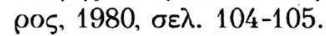

${ }^{25} \Gamma$. $\Sigma \alpha \rho \alpha \nu \tau \dot{\alpha} \rho \eta \zeta$, «La poesia...»,

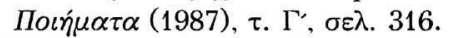

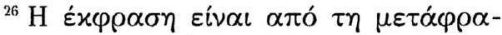

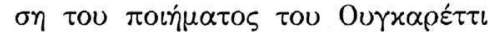

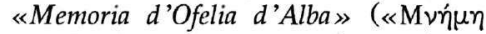

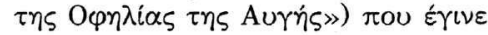
$\alpha \pi \delta$ to $\Sigma \alpha \rho \alpha \nu \tau \alpha ́ \rho \eta$.

${ }^{27}$ M. Petrucciani, Segnali e archetipi della poesia, Milano, Mursia, 1974, $\sigma \varepsilon \lambda .70$.

${ }^{28} \mathrm{G}$. Manacorda, «Giuseppe Ungaretti», Novecento, Bologna, Calderini, 1978, $\sigma \varepsilon \lambda .106$

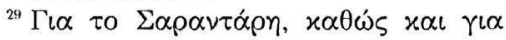

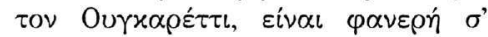

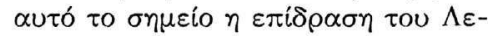
o $\pi \alpha ́ \rho \nu \tau l$.

${ }^{30}$ M. Petrucciani, Segnali e archetipi della poesia, ó. $\pi ., \sigma \varepsilon \lambda .71$.

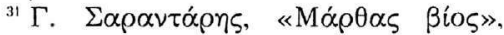

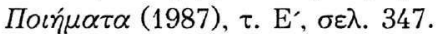

${ }^{32} \mathrm{G}$. Ungaretti, «Inno alla morte», Sentimento del tempo, Vita d'un uomo. Tutte le poesie, ó. $\pi$., $\sigma \varepsilon \lambda .117$.

${ }^{33} \mathrm{G}$. Ungaretti, «Memoria d'Ofelia D'Alba», Sentimento del tempo, Vita

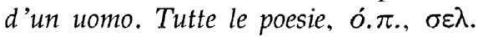

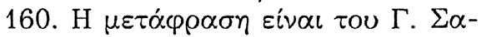
$\rho \alpha \nu \tau \alpha ́ \alpha \eta$.

${ }^{34}$ C. Ossola, Giuseppe Ungaretti, Milano,

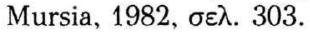

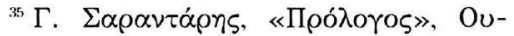

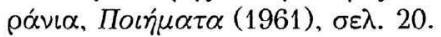

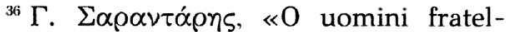

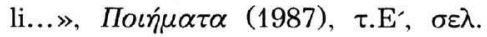
347.

${ }^{37} \mathrm{G}$. Ungaretti, «O notte», Sentimento del tempo, Vita d'un uomo. Tutte le poesie, ó. $\pi$., $\sigma \in \lambda .103$.

${ }^{38} \mathrm{G}$. Ungaretti, «Fratelli», L'Allegria, Vita d'un uomo. Tutte le poesie, ó. $\pi$., $\sigma \varepsilon \lambda .39$.

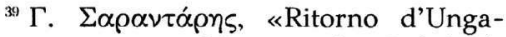

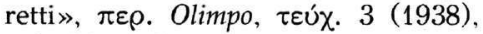
$\sigma \varepsilon \lambda .221-222$.

${ }^{40} \mathrm{G}$. Ungaretti, «Punto di mira», Vita d'un uomo. Saggi e interventi, Milano,

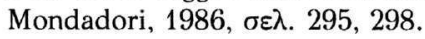

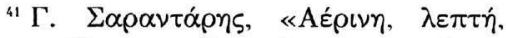

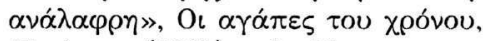

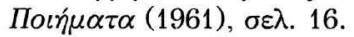

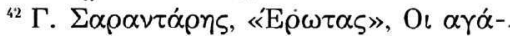

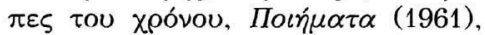
$\sigma \varepsilon \lambda .13$.

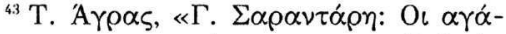

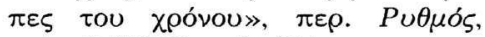

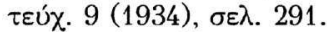

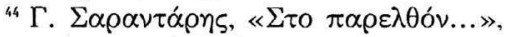

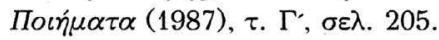

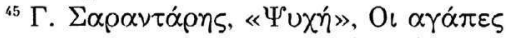

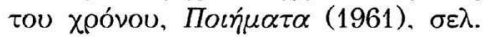
17.

${ }^{46} \mathrm{G}$. Ungaretti, «Girovago», L'Allegria, $V i t a$ d'un uomo. Tutte le poesie, ó. $\pi$., $\sigma \varepsilon \lambda .85$.

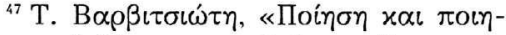

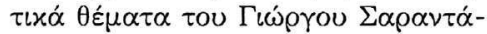

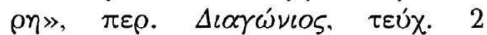
(1958), $\sigma \varepsilon \lambda .52$.

${ }^{48} \mathrm{~S}$. Ramat, Storia della poesia italiana del

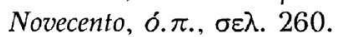

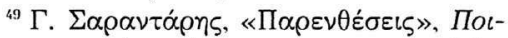
$\eta^{\prime} \mu \alpha \tau \alpha$ (1987), $\tau . \Gamma^{\prime}, \sigma \varepsilon \lambda .400$.

${ }^{50} \mathrm{G}$. Ungaretti, «Ragioni d'una poesia». $V i t a$ d'un uomo. Tutte le poesie, ó. $\pi$., $\sigma \varepsilon \lambda$. L XXII.

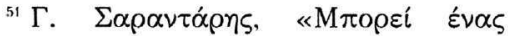

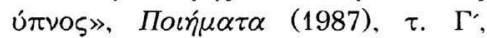
$\sigma \varepsilon \lambda .351$. 
${ }^{52}$ M. Petrucciani, Segnali e archetipi della poesia, ó. $\pi ., \sigma \varepsilon \lambda .68$.

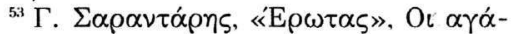

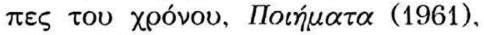
$\sigma \varepsilon \lambda .13$.

${ }^{54} \mathrm{G}$. Ungaretti, «Ti svelera», Sentimento del tempo, Vita d'un uomo. Tutte le poesie, ó. $\pi$., $\sigma \varepsilon \lambda .127$.

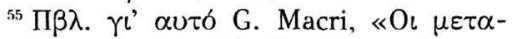

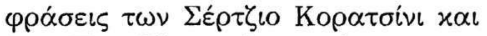

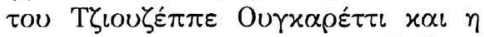

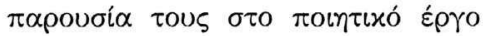

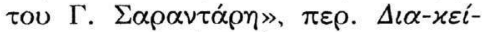

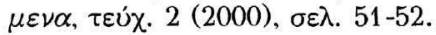

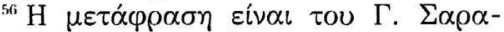

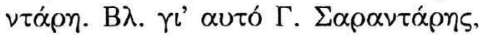

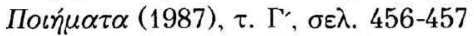

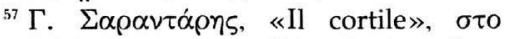
F.M. Pontani, Inediti italiani..., ó. $\pi$.,

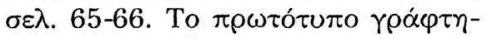
$x \varepsilon \sigma \tau \alpha \iota \tau \alpha \lambda \iota x \alpha$.

${ }^{58} \mathrm{G}$. Ungaretti, «Lucca», Vita d'un uomo. Tutte le poesie, ó. $\pi$. $\sigma \varepsilon \lambda$. 664665.

${ }^{59} \mathrm{G}$. Ungaretti, «Innocenza e memoria», Vita d'un uomo. Saggi e inter-

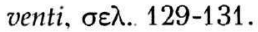

${ }^{60}$ M. Petrucciani, Segnali e archetipi della

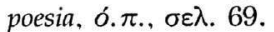

${ }^{61} \Gamma$. $\quad$ $\alpha \rho \alpha \nu \tau \dot{\alpha} \rho \eta \zeta, \quad \ll$ Sognare», $\sigma \tau 0$ 'F.M. Pontani, Inediti italiani..., ó. $\pi$., $\sigma \varepsilon \lambda .73-74$.

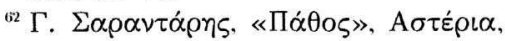
Поเท́ $\mu \alpha \tau \alpha$ (1961), $\sigma \varepsilon \lambda .32$.

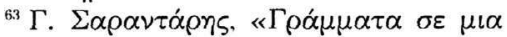
$\gamma u v \alpha i x \alpha »$, Пoiń $\mu \alpha \tau \alpha$ (1961), $\sigma \varepsilon \lambda$. 119.

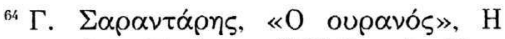

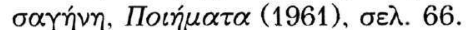

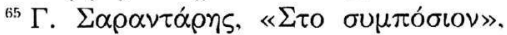

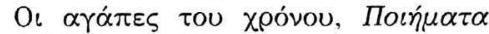

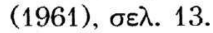

${ }^{66} \mathrm{G}$. Ungaretti, «O notte», Vita d'un

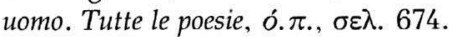

${ }^{67} \Gamma$. $\Sigma \alpha \rho \alpha \nu \tau \alpha \dot{\alpha} \emptyset$, «Appunti», Пoюn $\mu \alpha-$ $\tau \alpha$ (1987), $\tau . \mathrm{B}^{\prime}, \sigma \varepsilon \lambda .44$.
${ }^{68} \mathrm{~B} \lambda$. J. Chevalier - A. Gheerbrant. Dizionario dei simboli, Milano, Rizzoli. 1989, vol. 1, $\sigma \varepsilon \lambda .10 . \quad x \alpha \iota$ vol. 2 . $\sigma \varepsilon \lambda .67$.

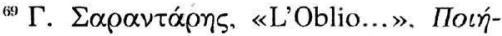
$\mu \alpha \tau \alpha$ (1987), $\tau$. B', $\sigma \varepsilon \lambda .38$.

${ }^{70} \mathrm{G}$. Ungaretti, «I fiumi», L'Allegria, Vita d'un uomo, ó. $\pi$. $\sigma \varepsilon \lambda .44$.

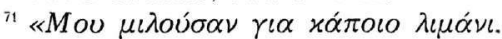

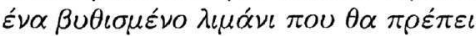

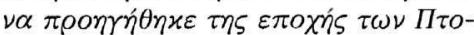

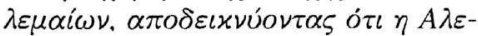

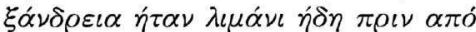

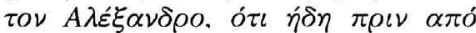

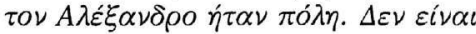

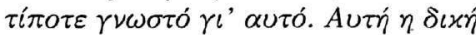

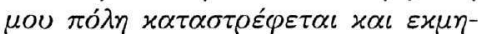

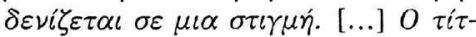

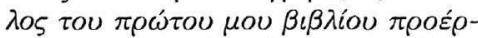

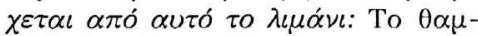

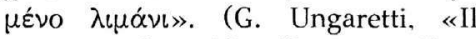
porto sepolto», Vita d'un uomo. Tutte le poesie, ó. $\pi$. $\sigma \varepsilon \lambda$. 520. To Porto

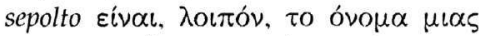

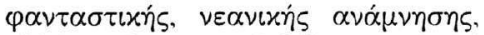

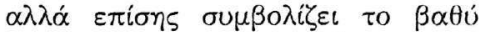

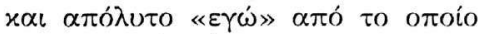

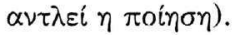

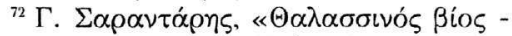

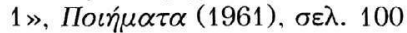

${ }^{73} \mathrm{G}$. Ungaretti, «Allegria di naufragi». L'Allegria, Vita d'un uomo. Tutte le poesie, ó. $\pi$., $\sigma \varepsilon \lambda .61$

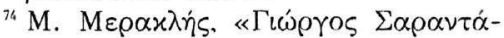

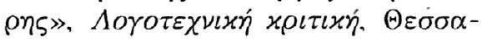

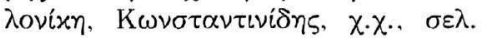
21.

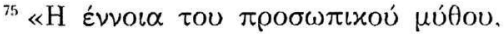

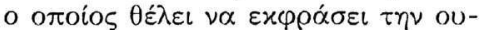

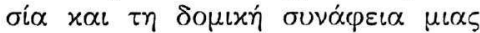

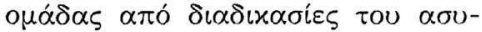

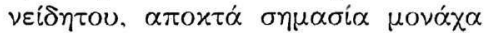

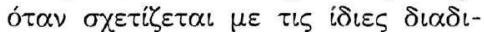

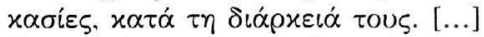

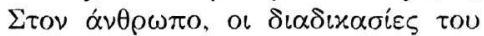

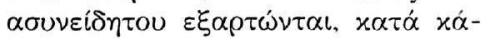




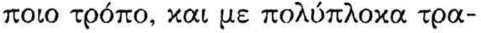

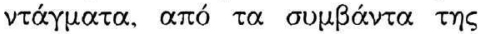

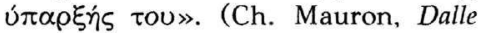
metafore ossessive al mito personale, Milano, Il Saggiatore, 1966, $\sigma \varepsilon \lambda$. 272-274)

${ }^{76} \mathrm{C}$. Ossola, Giuseppe Ungaretti, ó. $\pi$., $\sigma \varepsilon \lambda .234$.

${ }^{77} \mathrm{G}$. Ungaretti, «A riposo», L'Allegria, Vita d'un uomo. Tutte le poesie, ó. $\pi$., $\sigma \varepsilon \lambda .26$.

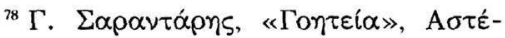

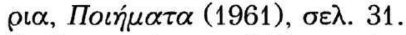

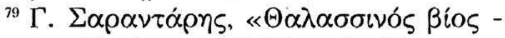

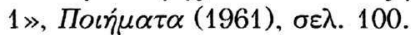

${ }^{80} \mathrm{G}$. Ungaretti, «Universo», L'Allegria, $V$ ita d'un uomo. Tutte le poesie, ó. $\pi$., $\sigma \varepsilon \lambda .49$.

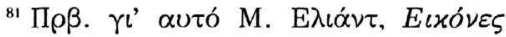

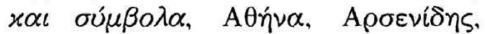

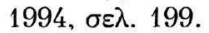

${ }^{82}$ C. Ossola, Giuseppe Ungaretti, ó. $\pi$., $\sigma \varepsilon \lambda .235$

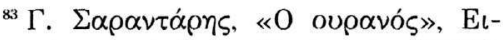

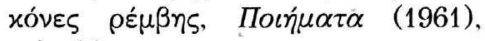
$\sigma \varepsilon \lambda .66$.

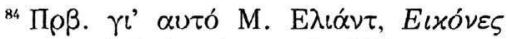

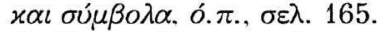

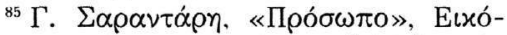

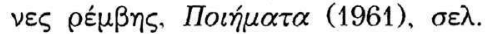
65.

${ }^{86} \mathrm{G}$. Ungaretti, «Popolo», L'Allegria, Vita d'un uomo. Tutte le poesie, ó. $\pi$., $\sigma \varepsilon \lambda .16$.

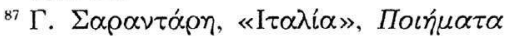

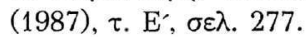

${ }^{88} \mathrm{G}$. Ungaretti, «Silenzio», L'Allegria, Vita d'un uomo. Tutte le poesie, ó. $\pi$., $\sigma \varepsilon \lambda .33$.

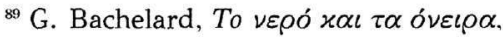
A $\theta \dot{\eta} \nu \alpha, \quad \mathrm{X} \alpha \tau \zeta \eta \nu เ x \circ \lambda \dot{\eta}, 1985, \quad \sigma \varepsilon \lambda$. 131.

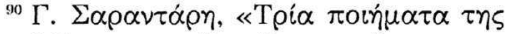

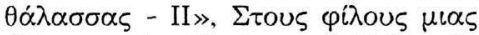

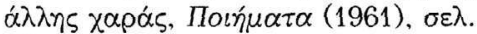
87.

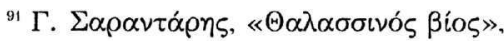

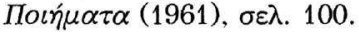

${ }^{92} \mathrm{G}$. Ungaretti, «La notte bella», L'Allegria, Vita d'un uomo. Tutte le poesie, ó. $\pi$., $\sigma \varepsilon \lambda .48$.

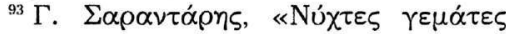

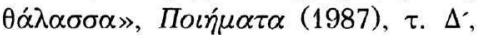
$\sigma \varepsilon \lambda .412$.

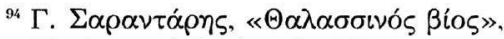
Поь́ं $\mu \alpha \tau \alpha$ (1961), $\sigma \varepsilon \lambda .105$

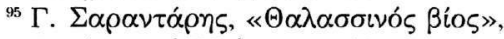
Поэท́ $\mu \alpha \tau \alpha$ (1961), $\sigma \varepsilon \lambda .100$.

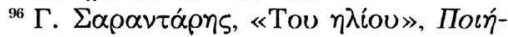
$\mu \alpha \tau \alpha$ (1987), $\tau . \Delta^{\prime}, \sigma \varepsilon \lambda .444$.

${ }^{97} \mathrm{G}$. Ungaretti, «A riposo», L'Allegria, Vita d'un uomo. Tutte le poesie, ó. $\pi$. $\sigma \varepsilon \lambda .26$.

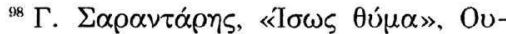

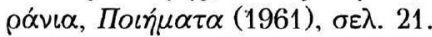

${ }^{99} \mathrm{G}$. Ungaretti, «Vanita», L'Allegria, Vita d'un uomo. Tutte le poesie, ó. $\pi$.,

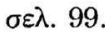

${ }^{100} \Pi \rho \beta$. G. Bachelard, To $\nu \varepsilon \rho o ́ ~ \varkappa \alpha \iota ~ \tau \alpha$

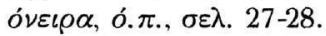

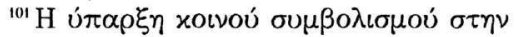

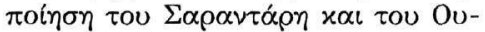

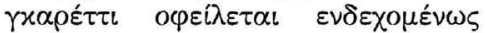

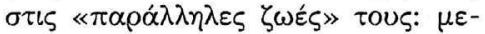

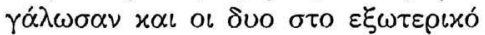
(o $\left.\Sigma \alpha \rho \alpha \nu \tau \alpha \alpha_{\eta}\right)_{5} \sigma \tau \eta \nu$ I $\tau \alpha \lambda i \alpha$, o Ov-

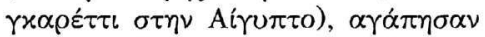

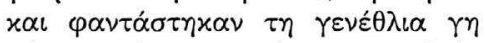

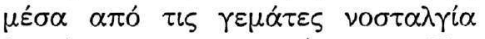

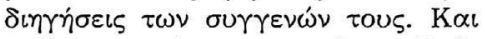

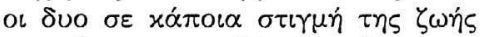

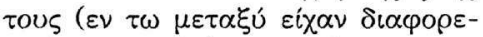

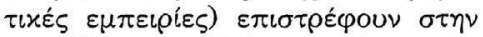

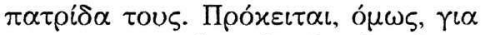

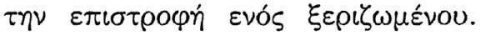

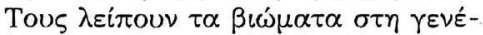

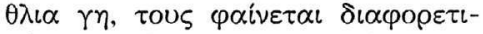

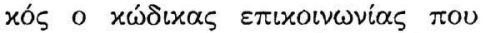

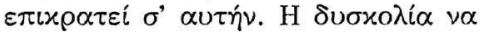

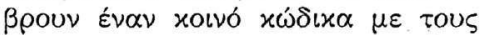

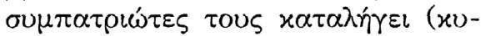

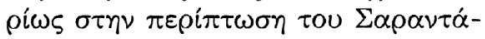




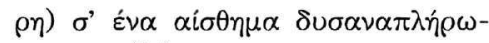

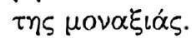

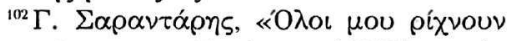

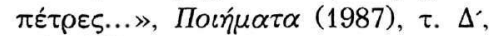
$\sigma \varepsilon \lambda .331$.

${ }^{103} \mathrm{G}$. Ungaretti, «Sempre notte», L'Allegria, Vita d'un uomo. Tutte le poesie, ó. $\pi$., $\sigma \varepsilon \lambda .71$.

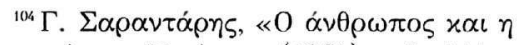

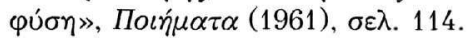

${ }^{1105} \mathrm{G}$. Ungaretti, «Sereno», L'Allegria, Vita d'un uomo. Tutte le poesie, ó. $\pi$., $\sigma \varepsilon \lambda .86$.

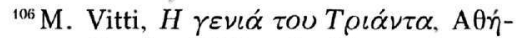

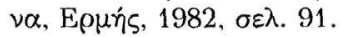

\section{Résumé}

Gabriella MACRì: Giorgos Sarandaris et Giuseppe Ungaretti: une approche comparative de leurs œuvres

I e poète grec Ghiorgos Sarandaris passa une grande partie de sa vie Len Italie. Il connaissait la langue italienne et pouvait lire, en italien, les recueils de poésie de Ungaretti, et même aussi ses essais. Dans l'essai «Ritorno di Ungaretti» de 1938 on dirait que Sarandaris reprend les théories que le poète italien développait dans son écrit de 1926 «Innocenza e memoria». Mais ce n’est pas seulement l'«élément technique» le trait d'union avec la poétique d'Ungaretti: Sarandaris avait un intérêt particulier pour le symbolisme de sa poésie (que le même poète italien expliquait souvent dans ses essais) et pour l'écriture hermétique, au point d'en faire un modèle pour sa recherche poétique, peut-être pour une identification de caractére autobiographique. L'adoption du symbolisme 'de l'eau et des autres archétypes (soleil, miroir), est, d'après moi, de la plus grande importance. 